\title{
Déterminants individuels et sociaux du test de dépistage du SRAS-CoV-2 et de l'obtention d'un résultat positif en Ontario, au Canada : une étude populationnelle
}

\author{
Maria E. Sundaram PhD, Andrew Calzavara MSc, Sharmistha Mishra MD PhD, Rafal Kustra PhD, \\ Adrienne K. Chan MD, Mackenzie A. Hamilton BSc, Mohamed Djebli BSc, Laura C. Rosella PhD, \\ Tristan Watson MSP, Hong Chen PhD, Branson Chen MSc, Stefan D. Baral MD, Jeffrey C. Kwong MD
}

Citation : CMAJ 2021 May 17;193:E723-34. doi : 10.1503/cmaj.202608-f; diffusion hâtive le 27 avril 2021

Voir la version anglaise de l'article ici : www.cmaj.ca/lookup/doi/10.1503/cmaj.202608

\section{Résumé}

Contexte : Optimiser la réponse de la santé publique pour diminuer le fardeau de la COVID-19 nécessite la caractérisation de l'hétérogénéité du risque posé par la maladie à l'échelle de la population. Cependant, l'hétérogénéité du dépistage du SRAS-CoV-2 peut fausser les estimations selon le modèle d'étude analytique utilisé. Notre objectif était d'explorer les biais collisionneurs dans le cadre d'une vaste étude portant sur les déterminants de la maladie et d'évaluer les déterminants individuels, environnementaux et sociaux du dépistage et du diagnostic du SRAS-CoV-2 parmi les résidents de l'Ontario, au Canada.

Méthodes : Nous avons exploré la présence potentielle de biais collisionneurs et caractérisé les déterminants individuels, environnementaux et sociaux de l'obtention d'un test de dépistage et d'un résultat positif à la présence de l'infection au SRAS-CoV-2 à l'aide d'analyses transversales parmi les 14,7 millions de personnes vivant dans la collectivité en Ontario, au Canada. Parmi les personnes ayant obtenu un diagnostic, nous avons utilisé des études analytiques distinctes afin de comparer les prédicteurs pour les personnes d'obtenir un résultat de test de dépistage positif plutôt que négatif, pour les personnes symptomatiques d'obtenir un résultat de test de dépistage positif plutôt que négatif et pour les personnes d'obtenir un résultat de test de dépistage positif plutôt que de ne pas obtenir un résultat positif (c.-à-d., obtenir un résultat de test de dépistage négatif ou ne pas obtenir de test de dépistage). Nos analyses comprennent des tests de dépistage réalisés entre le $1^{\text {er }}$ mars et le 20 juin 2020.

Résultats : Sur 14695579 personnes, nous avons constaté que 758691 d'entre elles ont passé un test de dépistage du SRAS-CoV-2, parmi lesquelles 25030 (3,3\%) ont obtenu un résultat positif. Plus la probabilité d'obtenir un test de dépistage s'éloignait de zéro, plus la variabilité généralement observée dans la probabilité d'un diagnostic était grande parmi les modèles d'études analytiques, particulièrement en ce qui a trait aux facteurs individuels. Nous avons constaté que la variabilité dans l'obtention d'un test de dépistage était moins importante en fonction des déterminants sociaux dans l'ensemble des études analytiques. Les facteurs tels que le fait d'habiter dans une région ayant une plus haute densité des ménages (rapport de cotes corrigé 1,86 ; intervalle de confiance [IC] à $95 \%$ 1,75-1,98), une plus grande proportion de travailleurs essentiels (rapport de cotes corrigé 1,58; IC à 95\% 1,48$1,69)$, une population atteignant un plus faible niveau de scolarité (rapport de cotes corrigé 1,33 ; IC à $95 \% 1,26-1,41$ ) et une plus grande proportion d'immigrants récents (rapport de cotes corrigé 1,10 ; IC à 95\% 1,05-1,15), étaient systématiquement corrélés à une probabilité plus importante d'obtenir un diagnostic de SRAS-CoV-2, peu importe le modèle d'étude analytique employé.

Interprétation : Lorsque la capacité de dépister est limitée, nos résultats suggèrent que les facteurs de risque peuvent être estimés plus adéquatement en utilisant des comparateurs populationnels plutôt que des comparateurs de résultat négatif au test de dépistage. Optimiser la lutte contre la COVID-19 nécessite des investissements dans des interventions structurelles déployées de façon suffisante et adaptées à l'hétérogénéité des déterminants sociaux du risque, dont le surpeuplement des ménages, l'occupation professionnelle et le racisme structurel. 
a propagation du SRAS-CoV-2, le virus causant la COVID19, a entraîné une pandémie présentant une hétérogénéité d'exposition et de risque de transmission ${ }^{1-4}$.

Une hétérogénéité des déterminants sociaux de la COVID-19 peut exister aux niveaux individuel et communautaire (p. ex., par la densité des ménages ${ }^{5-7}$ ). De plus, les déterminants sociaux de la santé, comme les obstacles à l'accès aux soins de santé, l'occupation professionnelle, le racisme structurel et la xénophobie, sont impliqués dans le risque posé par la COVID-198,9. Les déterminants environnementaux, tels que la pollution de l'air, peuvent aussi jouer un rôle, comme le démontrent les données; une plus grande pollution de l'air augmente le risque d'infection à d'autres virus respiratoires ${ }^{10,11}$ et d'évolution vers des formes graves de la COVID-19 ${ }^{12,13}$. Les facteurs environnementaux sont liés au racisme structurel (p. ex., en contexte d'un logement de mauvaise qualité) $)^{12,14}$.

Employer des données observationnelles pour cibler les facteurs de risque de la COVID-19 s'appuie sur le dépistage du SRAS-CoV-2, un service qui n'est pas déployé de façon uniforme ${ }^{15}$. Les différences dans l'offre de dépistage introduisent la possibilité de biais de sélection ${ }^{16,17}$, dont des biais collisionneurs ${ }^{17}$. Ceuxci peuvent être introduits dans les études épidémiologiques des facteurs de risque de la COVID-19 si les facteurs étudiés sont corrélés à l'acquisition d'une infection et à la probabilité d'obtenir un test de dépistage $\mathrm{e}^{17-19}$. Par exemple, les données révèlent que les personnes atteintes de diabète sont plus susceptibles de présenter une forme grave de la COVID-19 si elles sont infectées au SRAS-CoV-2 ${ }^{20,21}$. Ainsi, si elles sont infectées, ces personnes peuvent être plus susceptibles d'obtenir un test de dépistage et conséquemment, le diabète peut sembler associé à un diagnostic de COVID-19 dans les études portant sur les personnes ayant obtenu un test de dépistage du SRAS-CoV-2, même si le diabète n'est pas un facteur de risque de l'infection ${ }^{17}$. L'inverse peut aussi se produire avec les maladies respiratoires sous-jacentes (p. ex., l'asthme) qui présentent des symptômes comparables à ceux causés par le SRAS-CoV-2, menant à d'apparentes corrélations potentiellement « protectrices » avec la COVID-1922.

Nos objectifs étaient d'explorer les biais collisionneurs dans le cadre d'une vaste étude portant sur les déterminants de la COVID-19 et d'examiner les déterminants individuels, environnementaux et sociaux du dépistage et du diagnostic parmi les 14,7 millions de personnes résidant en Ontario, au Canada ${ }^{17}$.

\section{Méthodes}

\section{Conception de l'étude, conditions et population}

Nous avons réalisé une étude observationnelle à l'aide de données de population issues de bases de données de laboratoire et d'administration sanitaire en Ontario. Le système de santé ontarien offre un accès universel aux services des hôpitaux et des médecins ${ }^{23}$ et au dépistage en laboratoire ${ }^{24}$. Nous avons utilisé les données de personnes qui ont obtenu un test de dépistage entre le $1^{\text {er }}$ mars et le 20 juin 2020 afin de cibler les déterminants associés au dépistage et utilisé ensuite 3 modèles d'études analytiques pour isoler les déterminants associés à un résultat positif au test de dépistage du SRAS-CoV-2.
Sources des données, couplages et critères d'inclusion Nous avons ciblé des états de dépistage à l'aide des données du Système d'information de laboratoire de l'Ontario (SILO) et couplé ces renseignements à des ensembles de données pertinents liés à la santé qui comprennent des renseignements portant sur la démographie, le recours aux soins de santé et la région. Ces ensembles de données ont été couplés à l'aide de codes d'identification uniques et analysés par l'ICES (auparavant connu sous le nom d'Institute for Clinical Evaluative Sciences) ${ }^{25}$.

Le SILO a saisi environ $88 \%$ de tous les cas de SRAS-CoV-2 identifiés en laboratoire rapportés par la province au cours de la période de l'étude (calculé comme le nombre de cas identifiés dans SILO divisé par le nombre de cas rapportés par le tableau de bord de la COVID-19 en Ontario au cours de la même période). Les dossiers SILO comprennent des dates de collecte des échantillons, des résultats et une boîte de texte pour indiquer les symptômes que les fournisseurs de soins de santé ont remplie au moment de l'échantillonnage. Nous avons obtenu des renseignements démographiques et environnementaux aux niveaux individuel et régional de la Base de données sur les personnes inscrites (BDPI), de la Base de données sur les congés des patients (BDCP), de la Base de données sur les chirurgies d'un jour et du Système national d'information sur les soins ambulatoires de l'Institut canadien d'information sur la santé, du programme Assurancesanté de l'Ontario, du Système d'information ontarien sur la santé mentale, de la Cohorte de santé populationnelle et d'environnement de l'Ontario et du Recensement de la population du Canada de $2016^{26}$ (annexe 1, tableau supplémentaire 1, accessible en anglais au www.cmaj.ca/lookup/doi/10.1503/ cmaj.202608/tab-related-content).

Pour les personnes ayant subi plus d'un test de dépistage intégré au Système d'information de laboratoire de l'Ontario, nous avons utilisé le premier test de dépistage positif ou indéterminé, ou le premier test de dépistage négatif si tous les tests réalisés au cours de la période de l'étude étaient négatifs. Nous avons inclus les personnes qui n'ont pas subi de test de dépistage au cours de la période si elles n'étaient pas enregistrées comme étant décédées avant ou nées après le $1^{\mathrm{er}}$ mars 2020. Pour évaluer les déterminants d'obtention d'un test de dépistage et d'un diagnostic, nous avons intégré les personnes qui ont subi un test de dépistage d'amplification en chaîne par polymérase pour l'infection au SRAS-CoV-2 et qui ne résidaient pas dans des établissements de soins de longue durée au $1^{\text {er }}$ mars 2020 .

\section{Sélection et définition des déterminants potentiels de} résultats positifs à un test de dépistage du SRAS-CoV-2 Comme déterminants individuels, nous avons inclus le sexe, l'âge, les troubles de santé sous-jacents et le recours antérieur aux services de santé. Nous avons sélectionné les troubles de santé sous-jacents ciblés dans la documentation, révisée par des pairs ou non, comme étant associés à la gravité de la COVID192,27-30 ou avec des symptômes semblables à ceux de la COVID19, car la gravité et les symptômes peuvent mener à des écarts de dépistage et par conséquent, à un biais collisionneur ${ }^{31-36}$. Nous avons aussi sélectionné des problèmes de santé qui 
accroissent le besoin de soutien pour des soins personnels (p. ex., la démence), étant par conséquent le reflet d'une convergence avec les risques professionnels chez les fournisseurs de soins essentiels ${ }^{37,38}$.

Nous avons posé l'hypothèse que le recours aux soins de santé augmenterait l'accès aux tests de dépistage et serait un marqueur de comorbidités; nous avons mesuré le recours aux soins de santé par le nombre d'hospitalisations au cours des 3 dernières années, le nombre de consultations externes au cours de la dernière année et la vaccination contre la grippe au cours de la saison 2019-2020. Nous avons aussi intégré le groupe diagnostic corrigé $(G D C)^{40}$ du Johns Hopkins ACG System ${ }^{39}$ comme mesure composite des comorbidités.

Les déterminants environnementaux comprennent les particules fines $\left(\mathrm{PM}_{2,5}\right)$ à l'aide d'estimés obtenus par satellite ${ }^{41}$ et un modèle de régression d'aménagement du territoire pour $\mathrm{NO}_{2}^{42}$ au niveau du code postal.

Nous avons conceptualisé les déterminants sociaux comme des variables régionales qui pourraient signaler des taux de contact dans les communautés (densité des ménages, densité des immeubles d'appartements et statut des personnes ne vivant pas en couple, p. ex., non mariées) ${ }^{43,44}$, des taux de contact au travail ("travailleurs essentiels ») ${ }^{16,45}$, des barrières socioéconomiques à l'accès aux soins de santé ou au logement (revenu des ménages et niveau de scolarité) ${ }^{46,47}$ et des facteurs associés à la race et à l'origine ethnique (statut de minorité visible et immigration récente) $)^{8,9}$. Nous avons extrait ces variables du Recensement de la population du Canada de 2016 au niveau des aires de diffusion (AD), l'unité géographique la plus petite utilisée pour collecter les données du Recensement ${ }^{48}$. Nous avons classé les aires de diffusion à l'échelle des villes (pour un revenu médian équivalent par personne) ou à l'échelle de la province (pour tous les autres déterminants sociaux) puis nous les avons classées en quintiles. Pour la densité d'immeubles d'appartements et le statut d'immigration récente, la fréquence élevée de résultats nuls n'a permis la création que de 3 catégories (c.-à-d., une combinaison des 3 quintiles les moins élevés ainsi que le quatrième et cinquième quintile).

\section{Analyse statistique}

Nous avons défini le paramètre de test de dépistage comme l'obtention d'au moins 1 test de dépistage du SRAS-CoV-2 pendant la période de l'étude. Le groupe de comparaison comprend les résidents de l'Ontario qui n'ont pas eu de dossier de dépistage au cours de la période étudiée. Nous avons évalué les déterminants du dépistage dans des modèles de régression logistique non corrigés, corrigés en fonction de l'âge et du sexe et entièrement corrigés qui comprennent tous les déterminants. Le modèle entièrement corrigé comprend aussi une covariable à effet fixe pour la région sanitaire. Les régions sanitaires sont des zones géographiques au sein desquelles les mesures de santé publique ont été appliquées de manière différentes ${ }^{49}$ et où une variabilité des déterminants sociaux mesurés et non mesurés est possible ${ }^{50}$.

Afin de résoudre la présence potentielle de biais collisionneurs, nous avons comparé la probabilité d'obtenir un résultat positif à un test de dépistage du SRAS-CoV-2 extraite de modèles de régression logistique non corrigés, corrigés en fonction de l'âge et du sexe et entièrement corrigés (incluant tous les déterminants et les régions sanitaires) en utilisant 3 modèles d'études. Le modèle "pseudotest de dépistage négatif » comparait les personnes qui ont obtenu un résultat de test de dépistage positif aux personnes qui ont obtenu un résultat négatif, le modèle " vrai test de dépistage négatif » était limité aux personnes qui étaient enregistrées comme présentant des symptômes de la maladie ${ }^{51}$ et le modèle "cas-témoins » comparait toutes les personnes ayant obtenu un résultat de test de dépistage positif avec toutes les personnes n'ayant pas obtenu de résultats positifs (c.-à-d., les personnes présentant un résultat de test de dépistage négatif ou celle n'ayant pas obtenu de test de dépistage).

Pour cibler les déterminants des cas ayant obtenu un test de dépistage du SRAS-CoV-2 et ayant obtenu un résultat positif à la présence du virus dans le cadre d'un test de dépistage, nous nous sommes attardés aux résultats des modèles de régression logistique entièrement corrigés pour les études pseudotest de dépistage négatif et les études cas-témoins. Les résultats pour le modèle d'étude vrai test de dépistage négatif sont présentés à l'annexe 1 . Nous avons interprété chaque ensemble de déterminants comme des analyses indépendantes fondées sur des graphes orientés acycliques (annexe 1, figure supplémentaire 1 ). Nous sommes d'avis que le modèle d'étude cas-témoins est celui qui présente le moins de biais collisionneurs potentiels.

Nous avons réalisé l'analyse statistique à l'aide de la version 9.4 du SAS. Pour tenir compte de la colinéarité, nous avons évalué les tolérances et les facteurs d'inflation de la variance.

\section{Approbation éthique}

L'utilisation des données pour le présent projet a été autorisée en vertu de l'article 45 de la Loi de 2004 sur la protection des renseignements personnels sur la santé de l'Ontario, laquelle ne requière pas d'examen par un comité d'éthique de la recherche.

\section{Résultats}

Des 758691 personnes ayant subi un test de dépistage au cours de la période de l'étude, 25030 (3,3\%) ont obtenu un résultat positif au dépistage du SRAS-CoV-2 (figure 1). Seulement $11,8 \%$ des personnes testées présentaient un symptôme relevé par le fournisseur de soins, $13,6 \%$ étaient considérés comme asymptomatiques et les renseignements sur les symptômes étaient lacunaires pour 74,6 \% de ces personnes. Des caractéristiques descriptives de la population que nous avons étudiée sont regroupées au tableau 1 et à l'annexe 1, tableau supplémentaire 2 .

\section{Les déterminants pour le dépistage du SRAS-CoV-2}

Au cours de l'analyse entièrement corrigée, nous avons constaté que la probabilité de subir un test de dépistage augmentait avec l'âge (tableau 2 et annexe 1, tableau supplémentaire 3$)$. Les hommes présentaient une probabilité plus faible 


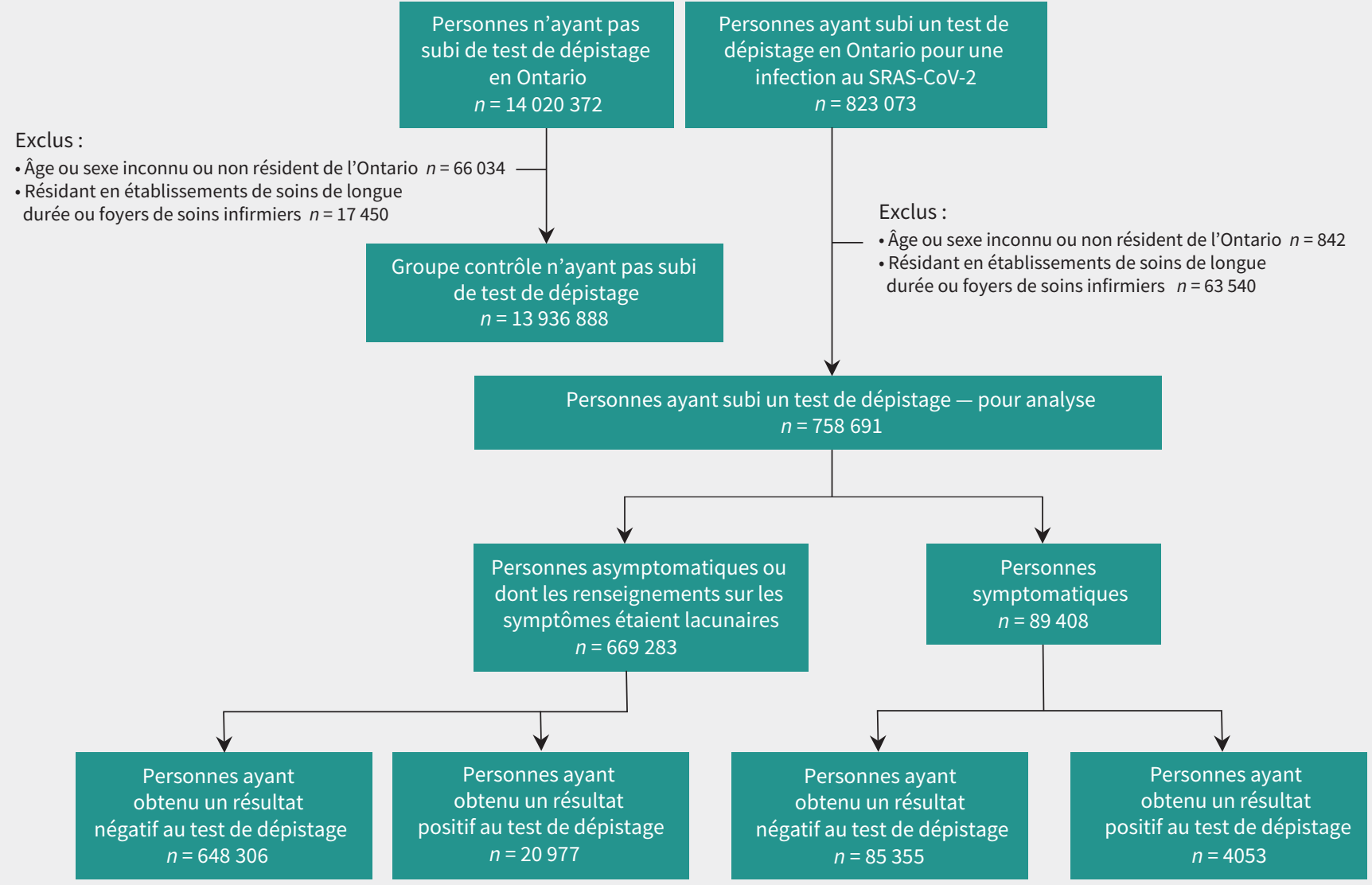

Figure 1 : Diagramme montrant les critères d'inclusion et d'exclusion ainsi que les ensembles de données analytiques résultants.

d'obtenir un test de dépistage que les femmes. Nous avons aussi constaté que presque tous les problèmes de santé sousjacents et la plupart des mesures de recours antérieurs aux services de santé étaient associés à une augmentation de la probabilité de subir un test de dépistage. Par contre, une plus grande pollution de l'air était corrélée à une plus faible probabilité d'obtenir un test de dépistage. Il y avait peu de variabilité dans la probabilité d'obtenir un test de dépistage selon les déterminants sociaux de la santé basés sur la région. Cependant, les régions comprenant un plus grand nombre de personnes issues des minorités visibles avaient une plus faible probabilité d'obtenir un test de dépistage alors que les régions où le revenu des ménages était plus élevé et où un plus grand pourcentage de la population ne vivait pas en couple présentaient une plus grande probabilité d'obtenir un test de dépistage. Les estimations de la probabilité de subir un test de dépistage pour la plupart des déterminants sociaux de la santé semblent progressivement atténuées lorsqu'on passe des modèles de régression non corrigés, aux modèles corrigés en fonction de l'âge et du sexe à ceux entièrement corrigés. Notamment, la corrélation entre le fait d'obtenir un test de dépistage et le quintile du revenu a changé de direction après la correction (figure 2 et annexe 1, tableau supplémentaire 3).
Variabilité des déterminants d'un résultat positif au test de dépistage du SRAS-CoV-2 pour l'ensemble des études analytiques

Notre comparaison des résultats en utilisant les différents modèles d'études analytiques a souligné d'importantes différences au niveau des déterminants individuels et des différences moindres pour ce qui a trait aux déterminants sociaux (tableau 2 et annexe 1, tableaux supplémentaires 4-6). Les variables qui étaient associées au test de dépistage tendaient à montrer différentes relations avec un résultat positif à la présence de SRAS-CoV-2 dans l'ensemble des modèles d'études. Par exemple, la probabilité corrigée d'obtenir un test de dépistage pour les adultes de 85 ans et plus comparativement à ceux plus jeunes de 5 ans était de 5,60 (intervalle de confiance [IC] à 95\% 5,47-5,73) et la probabilité corrigée d'obtenir un résultat positif d'infection au SRAS-CoV-2 était de 1,76 (IC à $95 \% 1,51-2,06$ ) avec un modèle pseudotest de dépistage négatif et 7,26 (IC à 95\% 6,23-8,46) pour un modèle d'étude cas-témoins (tableau 2). Certains problèmes de santé associés à une plus grande probabilité d'obtenir un test de dépistage, comme les problèmes respiratoires chroniques et les indicateurs d'un recours antérieur aux services de santé, apparaissent comme un effet protecteur contre l'obtention d'un résultat positif au test de dépistage lorsque le modèle pseudotest de 
dépistage négatif est employé, mais ne révèle aucune corrélation ou augmentation de la probabilité d'obtenir un résultat positif au test de dépistage lorsque le modèle d'étude cas-témoins est utilisé. Les résultats que nous avons obtenus avec le modèle vrai test de dépistage négatif étaient largement comparables aux résultats fournis par le modèle pseudotest de dépistage négatif avec des intervalles de confiance élargis, à l'exception de la probabilité d'obtenir un résultat positif qui était plus élevée chez les personnes plus âgées en employant le modèle vrai test de dépistage négatif comparativement au modèle pseudotest de dépistage négatif, et plus bas pour les quintiles supérieurs des travailleurs essentiels pour le modèle vrai test de dépistage négatif comparativement au pseudotest de dépistage négatif (annexe 1, tableaux supplémentaires 4 et 5).

Déterminants d'un résultat positif du test de dépistage au SRAS-CoV-2 en utilisant le modèle d'étude cas-témoins

En utilisant le modèle d'étude cas-témoins, nous avons constaté qu'un âge avancé, certaines comorbidités (p. ex., l'hypertension, le diabète, l'insuffisance cardiaque congestive, la démence, la

Tableau 1 (partie 1 de 3) : Caractéristiques de la population étudiée qui a subi un test de dépistage du SRAS-CoV-2 et de celle qui n'en a pas subi en Ontario (de $1^{\text {er }}$ mars au 20 juin 2020)

\begin{tabular}{|c|c|c|c|c|}
\hline \multirow[b]{2}{*}{ Caractéristiques } & \multicolumn{2}{|c|}{$\begin{array}{c}\mathrm{N}^{\text {bre }}(\%) \text { de personnes qui ont subi un } \\
\text { test de dépistage* }\end{array}$} & \multicolumn{2}{|c|}{$\mathbf{N}^{\text {bre }}(\%)$ de la population en Ontario } \\
\hline & $\begin{array}{l}\text { Résultat négatif } \\
\text { du test de } \\
\text { dépistage } \\
n=733661\end{array}$ & $\begin{array}{l}\text { Résultat positif } \\
\text { du test de } \\
\text { dépistage } \\
n=25030\end{array}$ & $\begin{array}{l}\text { Groupe contrôle } \\
\text { n'ayant pas subi } \\
\text { de test de } \\
\text { dépistage } \\
n=13936888\end{array}$ & $\begin{array}{c}\text { Groupe ayant subi } \\
\text { un test de } \\
\text { dépistage } \\
n=758691\end{array}$ \\
\hline \multicolumn{5}{|l|}{ Démographie } \\
\hline Âge, année; moyenne \pm écart-type & $49,52 \pm 20,99$ & $47,45 \pm 19,77$ & $40,31 \pm 22,91$ & $49,45 \pm 20,95$ \\
\hline Sexe masculin & $289769(39,5)$ & $11627(46,5)$ & $6930233(49,7)$ & $301396(39,7)$ \\
\hline Habitant en milieu rural ou dans une petite ville $\dagger$ & $82034(11,2)$ & $887(3,5)$ & $1390426(10,0)$ & $82921(10,9)$ \\
\hline \multicolumn{5}{|l|}{ Problème de santé chronique } \\
\hline Asthme & $136556(18,6)$ & $3887(15,5)$ & $2085118(15,0)$ & $140443(18,5)$ \\
\hline MPOC & $34261(4,7)$ & $715(2,9)$ & $249636(1,8)$ & $34976(4,6)$ \\
\hline Hypertension & $226111(30,8)$ & $7488(29,9)$ & $2836790(20,4)$ & $233599(30,8)$ \\
\hline Diabète & $108758(14,8)$ & $4268(17,1)$ & $1363667(9,8)$ & $113026(14,9)$ \\
\hline Insuffisance cardiaque congestive & $35947(4,9)$ & $983(3,9)$ & $225153(1,6)$ & $36930(4,9)$ \\
\hline Démence ou fragilité & $31804(4,3)$ & $1106(4,4)$ & $124295(0,9)$ & $32910(4,3)$ \\
\hline Cancert & $24333(3,3)$ & $484(1,9)$ & $213036(1,5)$ & $24817(3,3)$ \\
\hline Immunovulnérable§ & $13325(1,8)$ & $319(1,3)$ & $107824(0,8)$ & $13644(1,8)$ \\
\hline Maladie hépatique à un stade avancé & $9020(1,2)$ & $212(0,8)$ & $78747(0,6)$ & $9232(1,2)$ \\
\hline Cardiopathie ischémique & $33855(4,6)$ & $880(3,5)$ & $324682(2,3)$ & $34735(4,6)$ \\
\hline Accident vasculaire cérébral ischémique ou AITף & $13777(1,9)$ & $411(1,6)$ & $99503(0,7)$ & $14188(1,9)$ \\
\hline Schizophrénie ${ }^{\star \star}$ & $7526(1,0)$ & $220(0,9)$ & $64494(0,5)$ & $7746(1,0)$ \\
\hline Alcoolisme et toxicomani $e^{\star \star}$ & $22238(3,0)$ & $478(1,9)$ & $216156(1,6)$ & $22716(3,0)$ \\
\hline \multicolumn{5}{|l|}{ Recours aux soins de santé } \\
\hline \multicolumn{5}{|l|}{ Quintile du groupe diagnostic corrigé (GDC) } \\
\hline 1 (0 groupe diagnostic corrigé) & $31959(4,4)$ & $1571(6,3)$ & $1616852(11,6)$ & $33530(4,4)$ \\
\hline 2 (1-2 groupes diagnostics corrigés) & $101895(13,9)$ & $3601(14,4)$ & $3150969(22,6)$ & $105496(13,9)$ \\
\hline 3 (3-4 groupes diagnostics corrigés) & $149173(20,3)$ & $5101(20,4)$ & $3385602(24,3)$ & $154274(20,3)$ \\
\hline 4 (5-6 groupes diagnostics corrigés) & $150394(20,5)$ & $5115(20,4)$ & $2626403(18,8)$ & $155509(20,5)$ \\
\hline 5 (7-27 groupes diagnostics corrigés) & $300240(40,9)$ & $9642(38,5)$ & $3157062(22,7)$ & $309882(40,8)$ \\
\hline $\begin{array}{l}\text { Hospitalisations au cours des } 3 \text { dernières années, } \\
\text { moyenne } \pm \text { écart-type }\end{array}$ & $0,44 \pm 1,34$ & $0,33 \pm 1,21$ & $0,19 \pm 0,60$ & $0,44 \pm 1,34$ \\
\hline $\begin{array}{l}\text { Consultations externes cours de la dernière année, } \\
\text { moyenne } \pm \text { écart-type }\end{array}$ & $7,35 \pm 8,68$ & $7,06 \pm 8,33$ & $4,67 \pm 6,05$ & $7,34 \pm 8,67$ \\
\hline Vaccination contre la grippe (saison 2019-2020) & $213722(29,1)$ & $5547(22,2)$ & $2978472(21,4)$ & $219269(28,9)$ \\
\hline
\end{tabular}


Tableau 1 (partie 2 de 3) : Caractéristiques de la population étudiée qui a subi un test de dépistage du SRAS-CoV-2 et de celle qui n'en a pas subi en Ontario (de $1^{\text {er }}$ mars au 20 juin 2020)

\begin{tabular}{|c|c|c|c|c|}
\hline \multirow[b]{2}{*}{ Caractéristiques } & \multicolumn{2}{|c|}{$\begin{array}{c}\mathbf{N}^{\text {bre }}(\%) \text { de personnes qui ont subi un } \\
\text { test de dépistage* }\end{array}$} & \multicolumn{2}{|c|}{$N^{\text {bre }}(\%)$ de la population en Ontario } \\
\hline & $\begin{array}{l}\text { Résultat négatif } \\
\text { du test de } \\
\text { dépistage } \\
n=733661\end{array}$ & $\begin{array}{l}\text { Résultat positif } \\
\text { du test de } \\
\text { dépistage } \\
n=25030\end{array}$ & $\begin{array}{l}\text { Groupe contrôle } \\
\text { n'ayant pas subi } \\
\text { de test de } \\
\text { dépistage } \\
n=13936888\end{array}$ & $\begin{array}{c}\text { Groupe ayant su } \\
\text { un test de } \\
\text { dépistage } \\
n=758691\end{array}$ \\
\hline \multicolumn{5}{|c|}{ Déterminants environnementaux $† \dagger$} \\
\hline \multicolumn{5}{|l|}{$\mathrm{PM}_{2,5}\left(\mu \mathrm{g} / \mathrm{m}^{3}\right.$ par an) } \\
\hline $2 \grave{a}<6$ & $161300(22,0)$ & $1831(7,3)$ & $2481201(17,8)$ & $163131(21,5)$ \\
\hline 6 à $<7$ & $91134(12,4)$ & $1766(7,1)$ & $1555790(11,2)$ & $92900(12,2)$ \\
\hline 7 à $<8$ & $207966(28,3)$ & $8476(33,9)$ & $4450218(31,9)$ & $216442(28,5)$ \\
\hline 8 à $<9$ & $211861(28,9)$ & $11127(44,5)$ & $4258069(30,6)$ & $222988(29,4)$ \\
\hline$\geq 9$ & $59674(8,1)$ & $1747(7,0)$ & $1073665(7,7)$ & $61421(8,1)$ \\
\hline \multicolumn{5}{|c|}{$\mathrm{NO}_{2}$ (partie par milliard par an) } \\
\hline 0 à 6 & $328613(44,8)$ & $5237(20,9)$ & $5505976(39,5)$ & $333850(44,0)$ \\
\hline 6 à 8 & $170693(23,3)$ & $5599(22,4)$ & $3409506(24,5)$ & $176292(23,2)$ \\
\hline$\geq 8$ & $232629(31,7)$ & $14111(56,4)$ & $4903460(35,2)$ & $246740(32,5)$ \\
\hline \multicolumn{5}{|c|}{ Déterminant social de la santéł‡ (niveau régional) } \\
\hline \multicolumn{5}{|c|}{ Quintile de densité des ménages§§ } \\
\hline $1(0-2,1)$ & $162623(22,2)$ & $3639(14,5)$ & $2474391(17,8)$ & $166262(21,9)$ \\
\hline $2(2,2-2,4)$ & $140653(19,2)$ & $3104(12,4)$ & $2368013(17,0)$ & $143757(18,9)$ \\
\hline $3(2,5-2,6)$ & $104896(14,3)$ & $2721(10,9)$ & $1866317(13,4)$ & $107617(14,2)$ \\
\hline $4(2,7-3,0)$ & $166089(22,6)$ & $6321(25,3)$ & $3291097(23,6)$ & $172410(22,7)$ \\
\hline $5(3,1-5,7)$ & $152578(20,8)$ & $8929(35,7)$ & $3775489(27,1)$ & $161507(21,3)$ \\
\hline \multicolumn{5}{|c|}{ Catégorie de densité d'immeubles multilogements $₫$} \\
\hline $1(0 \%-7,3 \%)$ & $391477(53,4)$ & $12377(49,4)$ & $7994323(57,4)$ & $403854(53,2)$ \\
\hline $2(7,4 \%-37,7 \%)$ & $145108(19,8)$ & $3874(15,5)$ & $2478855(17,8)$ & $148982(19,6)$ \\
\hline $3(37,7 \%-104 \%)$ & $190244(25,9)$ & $8463(33,8)$ & $3301839(23,7)$ & $198707(26,2)$ \\
\hline \multicolumn{5}{|c|}{ Quintile des personnes ne vivant pas en couple $e^{\star \star \star}$} \\
\hline $1(11,2 \%-33,7 \%)$ & $150044(20,5)$ & $3539(14,1)$ & $3130907(22,5)$ & $153583(20,2)$ \\
\hline $2(33,7 \%-38,4 \%)$ & $128561(17,5)$ & $3915(15,6)$ & $2698847(19,4)$ & $132476(17,5)$ \\
\hline $3(38,5 \%-43,6 \%)$ & $127689(17,4)$ & $4632(18,5)$ & $2579005(18,5)$ & $132321(17,4)$ \\
\hline $4(43,6 \%-51,0 \%)$ & $145560(19,8)$ & $5591(22,3)$ & $2633710(18,9)$ & $151151(19,9)$ \\
\hline $5(51,0 \%-94,6 \%)$ & $174985(23,9)$ & $7037(28,1)$ & $2732838(19,6)$ & $182022(24,0)$ \\
\hline \multicolumn{5}{|c|}{ Statut de travailleur essentiel††† } \\
\hline $1(0 \%-32,5 \%)$ & $145517(19,8)$ & $3941(15,7)$ & $2969492(21,3)$ & $149458(19,7)$ \\
\hline $2(32,5 \%-42,3 \%)$ & $155189(21,2)$ & $5077(20,3)$ & $3084781(22,1)$ & $160266(21,1)$ \\
\hline $3(42,3 \%-49,8 \%)$ & $149589(20,4)$ & $4686(18,7)$ & $2717510(19,5)$ & $154275(20,3)$ \\
\hline $4(50,0 \%-57,5 \%)$ & $143965(19,6)$ & $5236(20,9)$ & $2615078(18,8)$ & $149201(19,7)$ \\
\hline $5(57,5 \%-114,3 \%)$ & $133601(18,2)$ & $5816(23,2)$ & $2399120(17,2)$ & $139417(18,4)$ \\
\hline \multicolumn{5}{|c|}{ Quintile du revenu des ménages $\ddagger \ddagger \ddagger$} \\
\hline 1 (revenu le plus faible) & $156320(21,3)$ & $7000(28,0)$ & $2679780(19,2)$ & $163320(21,5)$ \\
\hline 2 & $148687(20,3)$ & $5288(21,1)$ & $2698807(19,4)$ & $153975(20,3)$ \\
\hline 3 & $145317(19,8)$ & $5084(20,3)$ & $2791340(20,0)$ & $150401(19,8)$ \\
\hline 4 & $140352(19,1)$ & $4019(16,1)$ & $2809529(20,2)$ & $144371(19,0)$ \\
\hline 5 (revenu le plus élevé) & $138103(18,8)$ & $3419(13,7)$ & $2815238(20,2)$ & $141522(18,7)$ \\
\hline
\end{tabular}


Tableau 1 (partie 3 de 3) : Caractéristiques de la population étudiée qui a subi un test de dépistage du SRAS-CoV-2 et de celle qui n'en a pas subi en Ontario (de $1^{\text {er }}$ mars au 20 juin 2020)

\section{$\mathbf{N}^{\text {bre }}(\%)$ de personnes qui ont subi un test de dépistage*}

\begin{tabular}{|c|}
\hline Caractéristiques \\
\hline Quintile du niveau de scolarité§§§ \\
\hline $1(0,0 \%-4,1 \%)$ \\
\hline $2(4,1 \%-7,5 \%)$ \\
\hline $3(7,5 \%-11,4 \%)$ \\
\hline $4(11,4 \%-17,1 \%)$ \\
\hline $5(17,1 \%-94.3 \%)$ \\
\hline Quintile de minorité visibleฯฯฯ \\
\hline $1(0,0 \%-2,2 \%)$ \\
\hline $2(2,2 \%-7,5 \%)$ \\
\hline $3(7,5 \%-18,7 \%)$ \\
\hline $4(18,7 \%-43,5 \%)$ \\
\hline $5(43,5 \%-102 \%)$ \\
\hline
\end{tabular}

Catégorie d'immigration récente ${ }^{\star \star \star \star}$

$$
\begin{aligned}
& 1(0,0 \%-2,1 \%) \\
& 2(2,1 \%-4,7 \%) \\
& 3(4,7 \%-41,2 \%)
\end{aligned}
$$

Résultat négatif
du test de
dépistage
$n=733661$

Résultat positif
du test de
dépistage
$n=25030$

$\begin{array}{ll}144457(19,7) & 3903(15,6) \\ 154215(21,0) & 4477(17,9) \\ 151457(20,6) & 5052(20,2) \\ 148159(20,2) & 5314(21,2) \\ 129580(17,7) & 6010(24,0)\end{array}$

\begin{tabular}{lr}
$130912(17,8)$ & $1716(6,9)$ \\
\hline $137826(18,8)$ & $2233(8,9)$ \\
\hline $137744(18,8)$ & $3201(12,8)$ \\
$153503(20,9)$ & $5466(21,8)$ \\
$167893(22,9)$ & $12140(48,5)$ \\
\hline $401300(54,7)$ & $8271(33,0)$ \\
$146772(20,0)$ & $5409(21,6)$ \\
$174829(23,8)$ & $11018(44,0)$
\end{tabular}

$2926432(21,0)$

$148360(19,6)$

$2978444(21,4)$

$158692(20,9)$

$2888525(20,7)$

$2659544(19,1)$

$156509(20,6)$

$153473(20,2)$

$2333134(16,7)$

$135590(17,9)$

$2115641(15,2)$

$132628(17,5)$

$2255245(16,2)$

$140059(18,5)$

$2451335(17,6)$

$140945(18,6)$

$3023752(21,7)$

$158969(21,0)$

$3940245(28,3)$

$180033(23,7)$

Remarque : $A D$ = aire de diffusion, $\mathrm{AIT}=$ accident ischémique transitoire, $\mathrm{GDC}=$ Groupe diagnostic corrigé, $\mathrm{MPOC}$ = maladie pulmonaire obstructive chronique,

$\mathrm{PM}_{2,5}=$ particules fines.

*Sauf indication contraire.

†Nous définissons un milieu rural comme étant situé à l'extérieur de la zone de navette d'une ville dont la population est supérieure à 10000 personnes.

$\ddagger$ Nous comptons les personnes si elles ont obtenu un diagnostic au cours des 5 dernières années.

$\S$ Nous considérons que les personnes sont immunovulnérables si elles sont atteintes du VIH, ont subi une transplantation d'organe ou de moelle épinière ou présentent un autre problème d'immunodéficience.

ๆCette catégorie comprend les personnes qui ont subi un accident vasculaire cérébral ischémique ou un accident ischémique transitoire au cours des 20 dernières années.

${ }^{* *}$ Cette catégorie comprend les personnes qui ont obtenu un diagnostic au cours des 2 dernières années.

††Les valeurs de $\mathrm{PM}_{2,5}>12 \mu \mathrm{g} / \mathrm{m}^{3}$ ou de $\mathrm{NO}_{2}>53$ parties par milliard par année seraient associées à un risque accru d'autres maladies respiratoires ${ }^{52}$. Les valeurs de $\mathrm{PM}_{2,5}$ et de $\mathrm{NO}_{2}$ sont fournies au niveau du code postal et non celui de l'aire de diffusion.

¥†Toutes les variables de cette catégorie sont des variables propres aux régions au niveau de l'aire de diffusion du Recensement de la population du Canada de 2016.

$\S \S$ Fourchette de personnes par logement.

IףLes chiffres du Recensement sont arrondis de façon aléatoire au nombre le plus haut ou le plus bas divisible par 5 le plus près, ce qui entraîne certaines imprécisions mineures :

$7,3 \%$ représentent le $60^{\mathrm{e}}$ percentile.

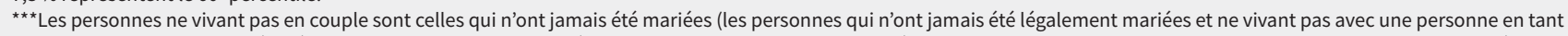
que couple); les personnes séparées (les personnes qui sont mariées, mais qui ne vivent plus avec leur époux [pour des raisons autres que, p. ex., la maladie, le travail ou les études], qui n'ont pas obtenu un divorce et ne vivant pas en couple avec une autre personne); les personnes divorcées (les personnes qui ont obtenu un divorce légal, qui ne se sont pas remariées et ne vivant pas en couple avec une autre personne) et les personnes veuves (les personnes qui ont perdu leur époux en raison d'un décès, qui ne se sont pas remariées et ne vivant pas en couple avec une autre personne).

†††Pourcentage des personnes de la région qui travaillent dans les domaines suivants : emplois dans le domaine de la vente et des services; emplois dans le domaine des métiers, du transport et de la machinerie et autres emplois associés; emplois dans le domaine des ressources naturelles, de l'agriculture et aux emplois de production associés; et emplois dans les domaines des industries manufacturières et des services publics. Les chiffres du Recensement sont arrondis de façon aléatoire au nombre le plus haut ou le plus bas divisible par 5 le plus près, ce qui entraîne certaines imprécisions mineures.

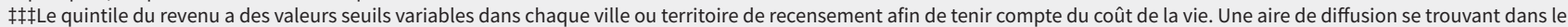
$1^{\text {er }}$ quintile signifie qu'elle se trouve dans le $20 \%$ inférieur des aires de diffusion de sa ville selon le revenu.

$\S \S \S$ Pourcentage des adultes de 25 à 64 ans de la région qui n'ont reçu aucun type de diplômes

๑ฯฯLe pourcentage des personnes de la région qui s'identifient comme une minorité visible. Les chiffres du Recensement sont arrondis de façon aléatoire au nombre le plus haut ou le plus bas divisible par 5 le plus près, ce qui entraîne certaines imprécisions mineures.

${ }_{\star \star \star \star *}$ Pourcentage des personnes de la région qui sont des immigrants récents; $2,1 \%$ représentent le $60^{\circ}$ percentile.

néphropathie chronique, l'accident vasculaire cérébral ischémique et l'accident ischémique transitoire) et l'augmentation du recours antérieur aux services de santé étaient associés à une augmentation de la probabilité d'obtenir un résultat positif au test de dépistage du SRAS-CoV-2. D’autres comor- bidités (c.-à-d., l'asthme, le cancer, la cardiopathie ischémique et la toxicomanie) et la vaccination contre la grippe au cours de la saison 2019-2020 étaient associées à une diminution de la probabilité d'obtenir un résultat positif au test de dépistage (tableau 2 et annexe 1, tableau supplémentaire 6). 
Tableau 2 (partie 1 de 3) : Probabilité d'obtenir un test de dépistage au SRAS-CoV-2 et un diagnostic de COVID-19 en Ontario entre le $1^{\text {er }}$ mars et le 20 juin 2020, selon des analyses corrigées employant 2 études analytiques

\begin{tabular}{|c|c|c|c|}
\hline \multirow[b]{2}{*}{ Déterminants } & \multirow{2}{*}{$\begin{array}{l}\text { Personnes ayant subi un test } \\
\text { de dépistage au SRAS-CoV-2 } \\
\text { Groupe ayant subi un test de } \\
\text { dépistage contre groupe } \\
\text { n'ayant pas subi de test de } \\
\text { dépistage } \\
\text { Rapport de cote corrigé } \\
\text { (IC à } 95 \% \text { ) }\end{array}$} & \multicolumn{2}{|c|}{ Personnes ayant obtenu un diagnostic de COVID-19 } \\
\hline & & $\begin{array}{l}\text { Nombre de tests positifs } \\
\text { contre nombre de tests } \\
\text { négatifs parmi toutes les } \\
\text { personnes testées } \dagger \\
\text { Rapport de cote corrigé } \\
\text { (IC à } 95 \%)\end{array}$ & $\begin{array}{l}\text { Groupe ayant obtenu } \\
\text { de dépistage positif ce } \\
\text { groupe n'ayant pas ob } \\
\text { un test de dépistage po } \\
\text { Rapport de cote corr } \\
\text { (IC à } 95 \%)\end{array}$ \\
\hline Taille de l'échantillon & 14695579 & 758691 & 14695579 \\
\hline \multicolumn{4}{|l|}{ Déterminants individuels } \\
\hline \multicolumn{4}{|l|}{ Groupe d'âge, ans (référence 0-4) } \\
\hline $5-19$ & $1,15(1,13-1,18)$ & $1,93(1,66-2,24)$ & $1,72(1,49-1,99)$ \\
\hline $20-34$ & $4,13(4,05-4,22)$ & $2,00(1,75-2,30)$ & $7,03(6,13-8,05)$ \\
\hline $35-49$ & $4,13(4,05-4,21)$ & $1,98(1,73-2,27)$ & $6,74(5,88-7,73)$ \\
\hline $50-64$ & $4,21(4,12-4,29)$ & $2,02(1,76-2,32)$ & $7,01(6,11-8,03)$ \\
\hline $65-74$ & $3,04(2,98-3,11)$ & $1,62(1,40-1,87)$ & $3,99(3,46-4,60)$ \\
\hline $75-84$ & $3,01(2,95-3,08)$ & $1,64(1,41-1,90)$ & $3,94(3,39-4,57)$ \\
\hline$\geq 85$ & $5,60(5,47-5,73)$ & $1,76(1,51-2,06)$ & $7,26(6,23-8,46)$ \\
\hline Sexe masculin & $0,76(0,76-0,76)$ & $1,26(1,23-1,30)$ & $1,00(0,98-1,03)$ \\
\hline $\begin{array}{l}\text { Habitant en milieu rural ou dans une petite } \\
\text { ville }\end{array}$ & $0,97(0,96-0,98)$ & $0,81(0,74-0,88)$ & $0,76(0,70-0,82)$ \\
\hline \multicolumn{4}{|l|}{ Problème de santé chronique sous-jacent } \\
\hline Asthme & $1,09(1,09-1,10)$ & $0,86(0,83-0,89)$ & $0,92(0,89-0,96)$ \\
\hline MPOC & $1,23(1,21-1,24)$ & $0,89(0,82-0,96)$ & $1,04(0,96-1,13)$ \\
\hline Hypertension & $0,98(0,98-0,99)$ & $1,12(1,08-1,16)$ & $1,13(1,09-1,17)$ \\
\hline Diabète & $0,98(0,97-0,99)$ & $1,26(1,21-1,31)$ & $1,19(1,14-1,23)$ \\
\hline Insuffisance cardiaque congestive & $1,26(1,24-1,28)$ & $0,99(0,92-1,07)$ & $1,25(1,16-1,35)$ \\
\hline Démence ou fragilité score $>15$ & $2,12(2,09-2,15)$ & $1,39(1,29-1,49)$ & $2,60(2,42-2,80)$ \\
\hline Cancer $\ddagger$ & $1,13(1,12-1,15)$ & $0,72(0,66-0,80)$ & $0,86(0,78-0,94)$ \\
\hline Maladie rénale chronique ${ }^{\star \star}$ & $1,31(1,29-1,32)$ & $0,86(0,80-0,93)$ & $1,16(1,08-1,24)$ \\
\hline Immunovulnérable†† & $1,30(1,28-1,33)$ & $0,79(0,70-0,89)$ & $1,02(0,91-1,14)$ \\
\hline Maladie hépatique à un stade avancé & $1,14(1,11-1,16)$ & $0,79(0,68-0,90)$ & $0,87(0,76-1,00)$ \\
\hline Cardiopathie ischémique & $1,02(1,01-1,03)$ & $0,88(0,82-0,95)$ & $0,90(0,84-0,97)$ \\
\hline $\begin{array}{l}\text { Accident vasculaire cérébral ischémique } \\
\text { ou AITł‡ }\end{array}$ & $1,15(1,13-1,17)$ & $1,05(0,95-1,17)$ & $1,18(1,06-1,31)$ \\
\hline Schizophrénie§§ & $1,24(1,21-1,27)$ & $0,84(0,73-0,97)$ & $0,99(0,87-1,13)$ \\
\hline Alcoolisme et toxicomanie§§ & $1,17(1,16-1,19)$ & $0,72(0,66-0,79)$ & $0,86(0,79-0,95)$ \\
\hline
\end{tabular}

\section{Recours aux soins de santé}

Quintile du groupe diagnostic corrigé (GDC) (Référence = 0 GDC)

$\begin{array}{ll}2 \text { (1-2 groupes diagnostics corrigés) } & 1,64(1,62-1,66) \\ 3 \text { (3-4 groupes diagnostics corrigés) } & 2,07(2,04-2,10) \\ 4 \text { (5-6 groupes diagnostics corrigés) } & 2,44(2,41-2,48) \\ 5 \text { (7-27 groupes diagnostics corrigés) } & 3,11(3,06-3,15)\end{array}$

$0,78(0,73-0,84)$

$1,24(1,17-1,32)$

$0,75(0,70-0,80)$

$1,46(1,37-1,56)$

$0,74(0,69-0,79)$

$1,69(1,57-1,81)$

$0,75(0,69-0,80)$

$2,12(1,97-2,28)$

Hospitalisations au cours des 3 dernières années (Référence $=0$ hospitalisation)

1

2

$\geq 3$
$0,99(0,98-1,00)$

$1,16(1,14-1,17)$

$1,75(1,73-1,78)$
$0,93(0,89-0,97)$

$0,86(0,79-0,93)$

$0,82(0,76-0,90)$
$0,92(0,88-0,96)$

$1,01(0,94-1,09)$

$1,38(1,27-1,50)$ 
Tableau 2 (partie 2 de 3) : Probabilité d'obtenir un test de dépistage au SRAS-CoV-2 et un diagnostic de COVID-19 en Ontario entre le $1^{\text {er }}$ mars et le 20 juin 2020, selon des analyses corrigées employant 2 études analytiques

Personnes ayant subi un test
de dépistage au SRAS-CoV-2

Groupe ayant subi un test de dépistage contre groupe

n'ayant pas subi de test de dépistage

Rapport de cote corrigé (IC à $95 \%)$

Personnes ayant obtenu un diagnostic de COVID-19*

Nombre de tests positifs contre nombre de tests négatifs parmi toutes les personnes testées $\dagger$ Rapport de cote corrigé (IC à $95 \%$ )
Groupe ayant obtenu un test de dépistage positif contre groupe n'ayant pas obtenu un test de dépistage positif $¥ \S$ Rapport de cote corrigé (IC à $95 \%$ )

Consultations externes au cours de la dernière année (Référence $=0-1$ consultation)

$\begin{array}{ll}2-4 & 1,08(1,07-1,09) \\ 5-8 & 1,11(1,10-1,12) \\ 9-14 & 1,17(1,16-1,18) \\ \geq 15 & 1,36(1,34-1,37) \\ \text { Vaccination contre la grippe (saison 2019-2020) } & 1,08(1,08-1,09)\end{array}$

$1,02(0,98-1,07)$
$1,03(0,98-1,08)$
$0,98(0,93-1,04)$
$0,91(0,85-0,97)$
$0,81(0,78-0,83)$

Déterminants environnementaux

Catégorie $\mathrm{PM}_{2,5}\left(\mu \mathrm{g} / \mathrm{m}^{3}\right)$ (Référence $=2-6$ )

$\begin{array}{ll}6-7 & 0,97(0,97-0,98) \\ 7-8 & 0,92(0,90-0,93) \\ 8-9 & 0,91(0,90-0,93) \\ \geq 10 & 0,90(0,89-0,92)\end{array}$

Catégorie $\mathrm{NO}_{2}$ (partie par milliard) (Référence $=0-6$ )

$$
\begin{array}{ll}
6-8 & 0,95(0,95-0,96) \\
\geq 8 & 0,94(0,93-0,95)
\end{array}
$$

\section{Déterminants sociaux de la santé ${ }^{\star \star \star}$ (niveau régional)}

Quintile de densité des ménages $† \dagger \dagger$ (Référence = premier quintile)

$\begin{array}{ll}2 & 1,01(1,00-1,02) \\ 3 & 1,03(1,02-1,04) \\ 4 & 1,01(1,00-1,02) \\ 5 & 0,97(0,95-0,98)\end{array}$

Catégorie de densité d'immeubles multilogementsłł‡ (Référence = première catégorie)

$\begin{array}{ll}2 & 1,04(1,03-1,04) \\ 3 & 1,01(1,00-1,02)\end{array}$

Quintile des personnes ne vivant pas en couple§§§ (Référence = premier quintile)

$\begin{array}{ll}2 & 1,02(1,01-1,03) \\ 3 & 1,07(1,06-1,08) \\ 4 & 1,19(1,18-1,21) \\ 5 & 1,39(1,38-1,41)\end{array}$

Catégorie de travailleur essentie|ฯฯฯ (Référence = première catégorie)

$\begin{array}{ll}2 & 1,04(1,03-1,04) \\ 3 & 1,06(1,05-1,07) \\ 4 & 1,05(1,04-1,06) \\ 5 & 1,04(1,03-1,06)\end{array}$

Quintile du revenu des ménages ${ }^{\star \star \star \star}$ (Référence $=$ premier quintile, revenu le plus bas)

$\begin{array}{ll}2 & 1,03(1,02-1,04) \\ 3 & 1,08(1,07-1,09) \\ 4 & 1,10(1,09-1,11) \\ 5 & 1,11(1,09-1,12)\end{array}$

$1,28(1,21-1,35)$

$\begin{array}{ll}0,91(0,85-0,99) & 0,92(0,85-0,99) \\ 1,10(0,99-1,21) & 1,00(0,91-1,10) \\ 1,29(1,16-1,43) & 1,19(1,08-1,32) \\ 1,45(1,29-1,63) & 1,31(1,16-1,47) \\ & \\ 1,05(1,00-1,11) & 1,00(0,96-1,06) \\ 1,13(1,06-1,21) & 1,05(0,98-1,12)\end{array}$

$1,21(1,14-1,27)$

$1,19(1,13-1,26)$

$1,39(1,31-1,48)$

$1,42(1,34-1,50)$

$1,70(1,61-1,79)$

$1,94(1,82-2,07)$

$1,70(1,61-1,80)$

$1,86(1,75-1,98)$

$1,00(0,95-1,04)$

$1,02(0,98-1,06)$

$1,18(1,12-1,24)$

$0,96(0,91-1,01)$

$0,97(0,92-1,02)$

$0,99(0,94-1,04)$

$1,11(1,05-1,17)$

$1,41(1,32-1,51)$

$1,25(1,19-1,32)$

$1,30(1,24-1,37)$

$1,37(1,30-1,45)$

$1,37(1,29-1,45)$

$1,51(1,42-1,60)$

$1,42(1,32-1,51)$

$1,58(1,48-1,69)$

$1,00(0,96-1,05)$

$1,12(1,06-1,18)$

$1,06(1,00-1,13)$

$1,07(0,99-1,15)$ 


\section{Personnes ayant subi un test} de dépistage au SRAS-CoV-2

\section{Groupe ayant subi un test de dépistage contre groupe n'ayant pas subi de test de dépistage Rapport de cote corrigé (IC à $95 \%$ )}

Personnes ayant obtenu un diagnostic de COVID-19*

Nombre de tests positifs
contre nombre de tests
négatifs parmi toutes les
personnes testéest
Rapport de cote corrigé
(IC à $95 \%$ )

Groupe ayant obtenu un test de dépistage positif contre groupe n'ayant pas obtenu Rapport de cote corrigé (IC à $95 \%$ )

Quintile du niveau de scolaritét††† (Référence = premier quintile)

$\begin{array}{ll}2 & 1,03(1,02-1,04) \\ 3 & 1,01(1,00-1,02) \\ 4 & 1,02(1,01-1,03) \\ 5 & 1,01(1,00-1,02)\end{array}$

$1,07(1,02-1,12)$
$1,15(1,09-1,21)$
$1,23(1,17-1,30)$
$1,37(1,29-1,46)$

$1,09(1,04-1,15)$

$1,15(1,09-1,21)$

$1,21(1,15-1,28)$

$1,33(1,26-1,41)$

Catégorie de minorité visiblełłł‡ (Référence = première catégorie)

2

3

4

5

Catégorie d’immigration récente§§§§ (Référence = première catégorie)
$0,99(0,98-1,00)$

$0,95(0,94-0,96)$

$0,91(0,90-0,92)$

$0,86(0,85-0,87)$

2

3

$0,99(0,99-1,00)$
$0,94(0,93-0,95)$

$$
\begin{aligned}
& 0,96(0,89-1,02) \\
& 0,97(0,91-1,05) \\
& 1,07(0,99-1,15) \\
& 1,27(1,17-1,38)
\end{aligned}
$$

$1,16(1,11-1,22)$

Remarque : $\mathrm{AIT}=$ accident ischémique transitoire, $\mathrm{GDC}=$ groupe diagnostic corrigé, $\mathrm{MPOC}=$ maladie pulmonaire obstructive chronique, $\mathrm{PM}{ }_{2,5}=$ particules fines, Référence = catégorie de référence.

*Tous les modèles présentés sont entièrement corrigés et comprennent l’ensemble des variables énumérées dans le présent tableau comme covariables.

†Étude de test pseudonégatif.

‡C'est-à-dire, tous les résultats de test négatifs et toutes les personnes n'ayant pas subi de test de dépistage.

§Étude cas-témoins.

INous définissons un milieu rural comme étant situé à l'extérieur de la zone de navette d'une ville dont la population est supérieure à 10000 personnes.

${ }^{\star *}$ Nous comptons les personnes si elles ont obtenu un diagnostic au cours des 5 dernières années.

††Nous considérons que les personnes sont immunovulnérables si elles sont atteintes du VIH, ont subi une transplantation d’organe ou de moelle épinière ou présentent un autre problème d'immunodéficience.

¥¥Cette catégorie comprend les personnes qui ont subi un accident vasculaire cérébral ischémique ou un accident ischémique transitoire au cours des 20 dernières années.

$\S \S$ Cette catégorie comprend les personnes qui ont obtenu un diagnostic au cours des 2 dernières années.

ฯฯ Les valeurs de $\mathrm{PM}_{2,5}>12 \mu \mathrm{g} / \mathrm{m}^{3}$ ou de $\mathrm{NO}_{2}>53$ parties par milliard par année serait associées à un risque accru d'autres maladies respiratoires ${ }^{52}$.

${ }^{\star \star \star}$ Toutes les variables de cette catégorie sont des variables propres aux régions au niveau de l'aire de diffusion (AD) du Recensement de la population du Canada de 2016

$\dagger \dagger \dagger$ Le premier quintile représente $0-2,1$ personnes par logement; deuxième quintile, 2,2-2,4 personnes par logement; troisième quintile, 2,5-2,6 personnes par logement; quatrième quintile, 2,7-3 personnes par logement et cinquième quintile, 3,1-5,7 personnes par logement.

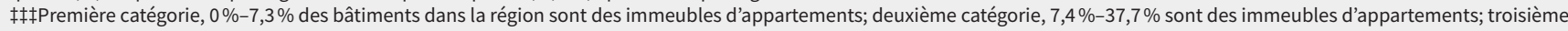
catégorie, $37,7 \%-100 \%$ sont des immeubles d'appartements.

§§§ Premier quintile, $11,2 \%-33,7 \%$ des personnes ne vivent pas en couple; deuxième quintile, $33,7 \%-38,4 \%$ des personnes; troisième quintile, $38,5 \%$ - $43,6 \%$ des personnes; quatrième quintile, $43,6 \%-51,0 \%$ des personnes et cinquième quintile, $51,0 \%-94,6 \%$ des personnes.

ฯฯฯ Le premier quintile représente $0 \%-32,5 \%$ des travailleurs de la région qui s'identifient comme travailleur essentiel; deuxième quintile, $32,5 \%-42,3 \%$ des personnes; troisième quintile, $42,3 \%-49,8 \%$ des personnes; quatrième quintile, $50,0 \%-57,5 \%$ des personnes et cinquième quintile, $57,5 \%-114,3 \%$.

$\star \star \star \star$ Le quintile du revenu a des valeurs seuils variables dans chaque ville ou territoire de recensement afin de tenir compte du coût de la vie. Une aire de diffusion se trouvant dans le $1^{\text {er }}$ quintile signifie qu'elle se trouve dans le $20 \%$ inférieur des aires de diffusion de sa ville selon le revenu.

t†††Le premier quintile, $0 \%-4,1 \%$ des personnes de 25 à 64 ans ne possédant pas de diplôme; deuxième quintile, 4,1\%-7,5\% des personnes; troisième quintile, 7,5\%-11,4\% des personnes; quatrième quintile, $11,4 \%-17,1 \%$ des personnes et cinquième quintile, $17,1 \%-94,3 \%$ des personnes.

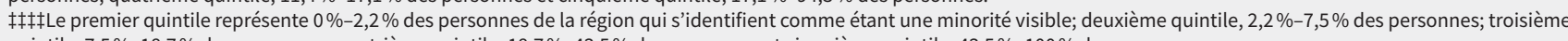
quintile, $7,5 \%-18,7 \%$ des personnes; quatrième quintile, $18,7 \%-43,5 \%$ des personnes et cinquième quintile, $43,5 \%-100 \%$ des personnes.

$\S \S \S \S L$ a première catégorie représente $0 \%-2,1 \%$ des personnes de l'aire de diffusion qui sont des immigrants récents; deuxième catégorie, $2,1 \%-4,7 \%$ des personnes et troisième catégorie, $4,7 \%-41,2 \%$ des personnes.

Les 2 catégories supérieures d'exposition au $\mathrm{PM}_{2,5}$ étaient associées à une augmentation de la probabilité d'obtenir un résultat positif, alors qu'aucune catégories de l'exposition au $\mathrm{NO}_{2}$ n'était associée à une telle augmentation.

Nous avons aussi constaté qu'une plus grande densité des ménages, une densité des immeubles d'appartements augmentée, un plus grand pourcentage de personnes ne vivant pas en couple et un pourcentage plus élevé de travailleurs essentiels étaient associés à une probabilité plus élevée d'obtenir un résultat positif au test de dépistage du SRAS-CoV-2. Un plus faible niveau de scolarité était relié à une augmentation de la probabilité, mais il n'existait pas de relation statistiquement cohérente avec le revenu des ménages. Nous avons aussi déterminé qu'être dans le quintile le plus élevé des milieux où vivent des personnes 
Patients ayant subi un test de dépistage

de l'infection au SRAS-CoV-2

$(n=758691)$

Nombre de personnes par logement (Référence $1^{\mathrm{er}}$ quintile)

Quintile 2

Quintile 3

Quintile 4

Quintile 5

Immeuble d'appartements (Référence ${ }^{\text {er }}$ tertile)

Tertile 2

Tertile 3

Nombre de personnes ne vivant pas en couple (Référence $1^{\text {er }}$ quintile)

Quintile 2

Quintile 3

Quintile 4

Quintile 5

Statut de travailleur essentiel (Référence $1^{\text {er }}$ quintile)

Quintile 2

Quintile 3

Quintile 4

Quintile 5

Revenu médian des ménages (Référence $1^{\mathrm{er}}$ quintile)

Quintile 2

Quintile 3

Quintile 4

Quintile 5

Niveau de scolarité (Référence $1^{\text {er }}$ quintile)

Quintile 2

Quintile 3

Quintile 4

Quintile 5

Pourcentage de minorités visibles (Référence $1^{\text {er }}$ quintile)

Quintile 2

Quintile 3

Quintile 4

Quintile 5

Pourcentage de nouveaux immigrants (Référence $1^{\mathrm{er}}$ tertile)

Tertile 2

Tertile 3
Modèle non corrigé

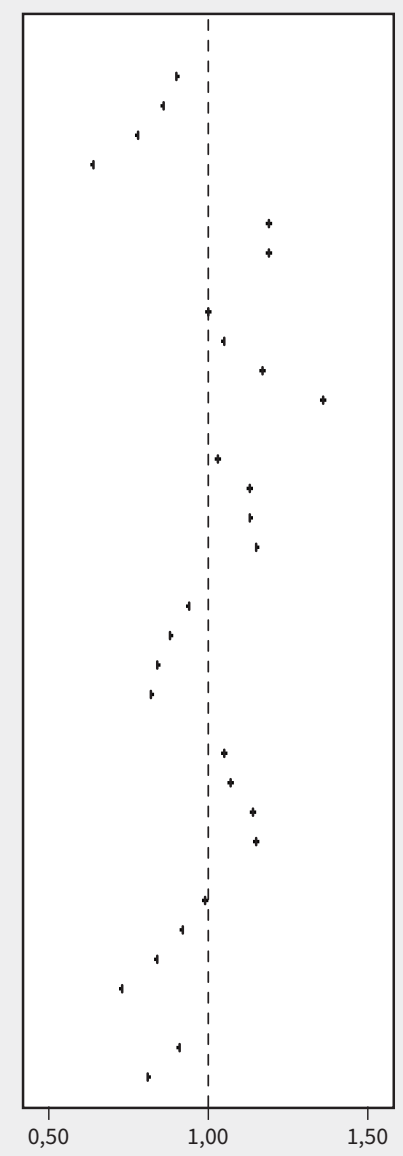

Modèle entièrement corrigé

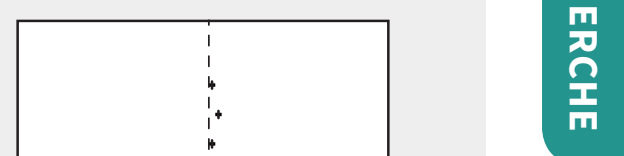

Rapport de cotes

Figure 2 : Corrélations non corrigée et entièrement corrigée entre les déterminants sociaux de la santé et le dépistage du SRAS-CoV-2 en Ontario (du $1^{\text {er }}$ mars au 20 juin 2020) en employant une étude cas-témoins. Les variables de cette figure sont énumérées comme des covariables et décrites au tableau 2. Remarque : Référence = catégorie de référence.

issues des minorités visibles et un plus grand pourcentage d'immigrants récents étaient corrélé à une plus grande probabilité d'obtenir un résultat positif au test de dépistage du SRASCoV-2. Les associations étaient atténuées après la correction pour tous les déterminants sociaux à l'exception de la densité des ménages et la présence de travailleurs essentiels (figure 3 et annexe 1 , tableau supplémentaire 6).

Notre évaluation de la colinéarité des diagnostics a révélé que toutes les tolérances étaient inférieures à 1 et que tous les facteurs d'inflation de la variance étaient inférieurs à 5 (annexe 1 , tableau supplémentaire 7 ).

\section{Interprétation}

Nous avons constaté que nos 3 modèles analytiques ciblaient différents déterminants individuels de l'obtention d'un résultat positif au test de dépistage du SRAS-CoV-2, probablement en rai- son de biais collisionneurs. En employant l'analyse cas-témoins, que nous considérons comme la moins partiale, nous avons ciblé des déterminants individuels, environnementaux et sociaux de la santé particuliers comme étant des déterminants clés de l'obtention d'un résultat positif au test de dépistage du SRAS-CoV-2.

En utilisant les modèles vrai test de dépistage négatif et pseudotest de dépistage négatif, nous avons constaté un haut potentiel de cibler de façon erronée des déterminants individuels, comme des problèmes de santé sous-jacents ayant un effet protecteur contre l'obtention d'un résultat positif au test de dépistage du SRAS-CoV-2, bien qu'ils soient associés à des taux plus élevés de subir un test de dépistage. Ces problèmes de santé sont associés à la gravité de la COVID-19² et peuvent être susceptibles au biais collisionneur, où la direction de l'effet mesuré change selon le modèle choisi. Des résultats comparables ont été constatés avec les 
Nombre de personnes par logement (Référence $1^{\text {er }}$ quintile) Quintile 2

Quintile 3

Quintile 4

Quintile 5

Immeuble d'appartements (Référence $1^{\mathrm{er}}$ tertile)

Tertile 2

Tertile3

Nombre de personnes ne vivant pas en couple (Référence $1^{\mathrm{er}}$ quintile)

Quintile 2

Quintile 3

Quintile 4

Quintile 5

Statut de travailleur essentiel (Référence $1^{\text {er }}$ quintile)

Quintile 2

Quintile 3

Quintile 4

Quintile 5

Revenu médian des ménages (Référence $1^{\text {er }}$ quintile)

Quintile 2

Quintile 3

Quintile 4

Quintile 5

Niveau de scolarité (Référence $1^{\text {er }}$ quintile)

Quintile 2

Quintile 3

Quintile 4

Quintile 5

Pourcentage de minorités visibles (Référence $1^{\mathrm{er}}$ quintile)

Quintile 2

Quintile 3

Quintile 4

Quintile 5

Pourcentage de nouveaux immigrants (Référence $1^{\text {er }}$ tertile)

Tertile 2

Tertile 3
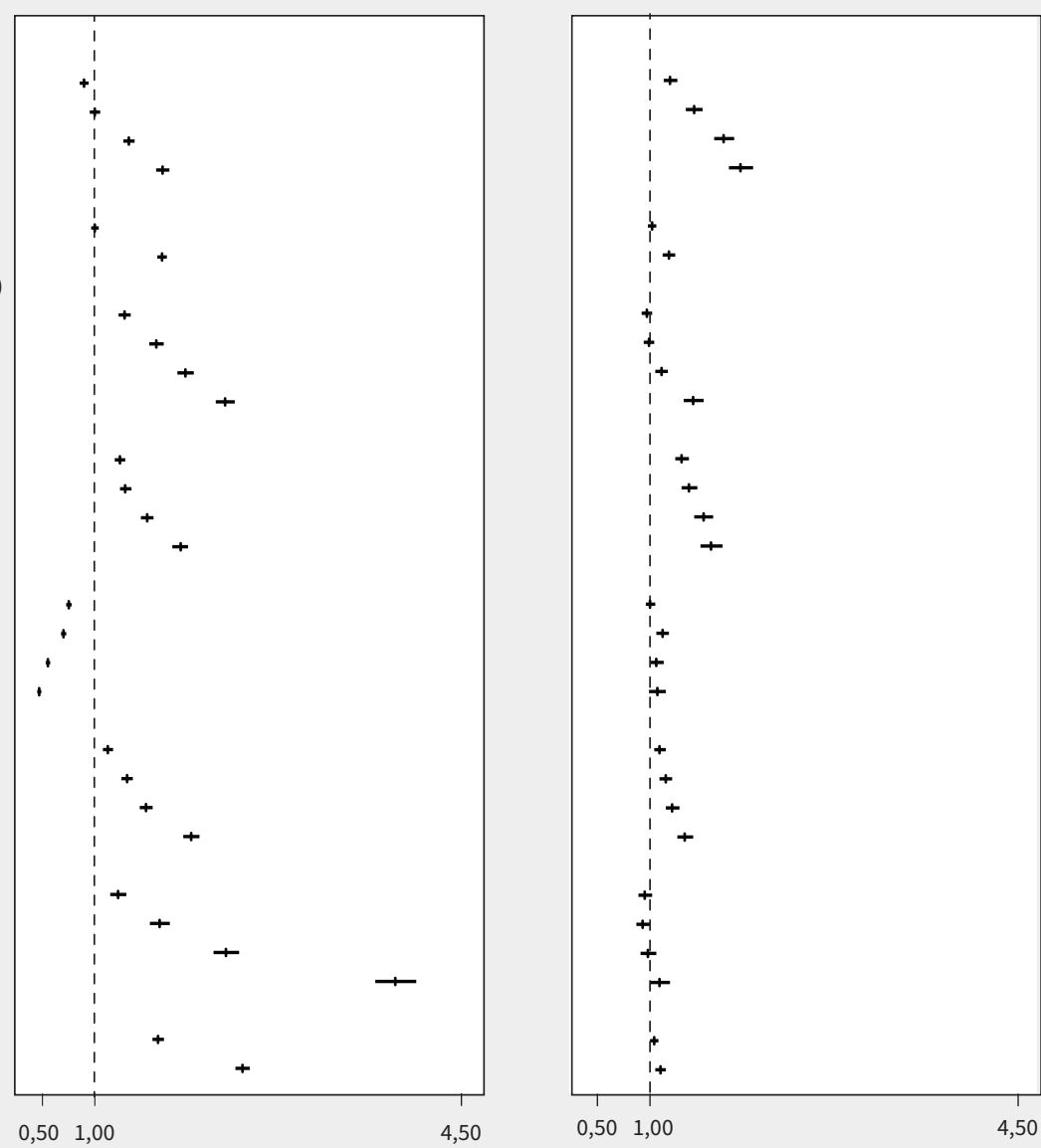

Rapport de cotes

Figure 3 : Corrélations non corrigée et entièrement corrigée entre les déterminants sociaux de la santé et un résultat positif au test de dépistage du SRAS-CoV-2 en Ontario (du $1^{\mathrm{er}}$ mars au 20 juin 2020) en employant une étude cas-témoins. Les variables de cette figure sont énumérées comme des covariables et décrites au tableau 2. Remarque : Référence = catégorie de référence.

variables de recours aux soins de santé. Ainsi, l'évaluation des déterminants de l'obtention d'un résultat positif au test de dépistage du SRAS-CoV-2 requiert une interprétation nuancée en évaluant les raisons du dépistage ${ }^{17}$. Dans le contexte du faible niveau de prestation des tests de dépistage, le modèle d'étude cas-témoins semble avoir atténué certaines sources potentielles de biais collisionneurs, dans l'hypothèse que le nombre de personnes non testées est comparable à celui du nombre de personnes qui ont obtenu un résultat négatif au test de dépistage ${ }^{16,17}$.

Nous avons constaté que certains problèmes de santé sousjacents demeuraient associés à des diagnostics lorsque le modèle d'étude cas-témoins est employé, étant le reflet de facteurs de confusion non mesurés ou d'une possible susceptibilité biologique à l'infection si exposé ${ }^{10,11,20,53,54}$. Par exemple, la démence et la fragilité demeuraient associées de façon indépendante au diagnostic, ce qui peut être causé par des facteurs de confusion non mesurés, comme les taux plus élevés de contacts avec les personnes qui les soignent ou le fait d'habiter dans des milieux de vie collectifs comme les résidences pour aînés. Ainsi, les problèmes de santé sous-jacents, comme la démence et la fragilité, représentent des cibles de prévention accompagnées de stratégies conçues sur mesure pour réduire l'exposition des personnes caractérisées par ces déterminants individuels.

Au cours de la période de l'étude, les critères de dépistage du SRAS-CoV-2 en Ontario sont passés d'une attention particulière portée aux voyageurs symptomatiques de retour au pays aux personnes présentant des symptômes graves et aux personnes exposées dans le cadre de leur emploi à davantage de tests de dépistage destinés aux personnes asymptômatiques ${ }^{31-34,36}$. Ces changements peuvent avoir engendré des différences entre les personnes qui ont subi un test de dépistage et qui présentaient des symptômes comparativement à l'ensemble des personnes qui étaient testées. Dans le cadre de notre étude, la restriction du modèle de test de dépistage négatif aux personnes présentant 
des symptômes n'a pas fourni des résultats nettement différents du modèle de test de dépistage négatif qui tenait compte des personnes symptomatiques et asymptomatiques pour la majorité des déterminants, mais ce peut être causé en partie par la forte proportion de personnes dont les renseignements sur les symptômes étaient lacunaires (74,6\%).

La corrélation indépendante entre un $\mathrm{PM}_{2,5}$ élevé et un diagnostic peut être le reflet de déterminants sociaux de la santé non mesurés ${ }^{55,56}$. Cependant, des études ont aussi invoqué la pollution environnementale comme ayant une influence sur le risque d'exposition au SRAS-CoV-2 et la gravité de la COVID-19 $10-12$.

Nous avons observé une probabilité accrue d'obtenir un résultat positif à un test de dépistage du SRAS-CoV-2 lorsqu'associée à la densité des ménages, au pourcentage d'immeubles d'appartements, au nombre de personnes ne vivant pas en couple, aux travailleurs essentiels, au niveau de scolarité et au statut d'immigration récente, le tout cohérent avec les constats d'autres milieux ${ }^{50,57,58}$. On a démontré que la taille des ménages est un facteur de risque cohérent sur un vaste éventail de milieux ${ }^{59,60}$. Ces taux d'infection plus élevés sont vraisemblablement causés par des contacts rapprochés et prolongés entre les personnes qui se produisent plus fréquemment au sein des ménages ${ }^{60}$. Les travailleurs des services essentiels sont aussi associés à un risque d'exposition plus élevé ${ }^{61}$, que ce soit parce que ces emplois ne peuvent être réalisés avec les méthodes de protection appropriées ou que les politiques et le matériel de protection ne sont pas mis en place, exposant les travailleurs à un risque élevé62,63.

Nous avons constaté que des pourcentages plus élevés d'immigrants récents dans une région étaient associés à une probabilité accrue d'obtenir un résultat positif à un test de dépistage du SRAS-CoV-2, même après avoir procédé aux corrections, bien que le pourcentage des minorités visibles ne l'était pas. Ces deux variables pourraient représenter des mesures résiduelles de racisme structurel, potentialisant le risque accru d'exposition au SRAS-CoV-2 et à la gravité de la COVID-1964-66, y compris l'hospitalisation et le décès liés à la COVID-199,16,28,58. Nous avons constaté que la corrélation entre le statut de minorité visible et le diagnostic était atténuée après la correction pour les déterminants individuels et environnementaux, de même que d'autres déterminants sociaux de la santé. Ces constats sont vraisemblablement le reflet de ce que nous savons déjà au sujet des la race et de l'ethnicité en tant que constructions sociales et déterminants sociaux de la santé ${ }^{67}$. Enfin, le fait qu'il y avait peu de corrélation entre la plupart des déterminants sociaux de la santé et la probabilité d'obtenir un test de dépistage tend à montrer que les ressources de dépistage n'étaient peut-être pas priorisées de façon adéquate pour les personnes à plus haut risque ${ }^{68}$.

Nos résultats tendent à montrer le besoin d'augmenter et de rediriger les ressources qui visent spécifiquement les déterminants sociaux comme la densité des ménages ${ }^{47,69}$ (p. ex., des centres d'isolation volontaire ${ }^{70}$ et les services complets $\left.{ }^{71}\right)$, les risques professionnels ${ }^{62,66}$ ( $p$. ex., des congés de maladie payés $^{72}$, le dépistage en milieu de travail ${ }^{73}$ et l'amélioration de la ventilation ${ }^{62}$ ) et d'autres médiateurs du racisme structurel ${ }^{68,74,75}$ (p. ex., des campagnes de sensibilisation au dépistage menées par la communauté $\left.{ }^{76}\right)$. Ils suggèrent aussi de prioriser les stratégies de vaccination contre la COVID-19 visant les communautés et les milieux de travail qui présentent les plus hauts taux de transmission. Bien que l'administratrice en chef de la santé publique du Canada ait suggéré que la réponse de la santé publique à la COVID-19 soit fondée sur l'équité ${ }^{45}$, la plupart des mesures d'équité contre la COVID19 et de sensibilisation auprès des communautés marginalisées ont été mises en œuvre par des petits groupes indépendant, dont des organismes bénévoles ${ }^{77-79}$.

\section{Limites de l'étude}

Notre détermination des résultats positifs au test de dépistage du SRAS-CoV-2 était limitée aux cas confirmés en laboratoire et aux $88 \%$ de tous les diagnostics provinciaux qui étaient accessibles par SILO. Nous avons supposé que les déterminants demeuraient constants tout au long de la période étudiée alors que les données de surveillance suggèrent une évolution dans la manière dont les infections se propagent entre les réseaux sociaux ${ }^{80}$. De futures analyses devraient évaluer les changements dans la direction et l'ampleur des déterminants tout au long de l'éclosion. Nos modèles étaient corrigés aussi selon la région de santé publique au sein de laquelle plusieurs déterminants sociaux sont regroupés ${ }^{50}$ et nous ne pouvons pas déduire de nos résultats la manière dont les déterminants sociaux des diagnostics peuvent varier entre les régions géographiques et au sein de ces régions. Nous avons mesuré les déterminants sociaux au niveau régional, mais ces déterminants n'étaient pas accessibles au niveau individuel; cependant, en décrivant les voisinages des individus, notre analyse reflète le rôle des déterminants structurel et environnemental pour les personnes qui y résident. Il se peut que nous ayons surcorrigé les modèles entièrement corrigés dans nos analyses en raison de nombre élevé de covariables. Cependant, la direction des estimations d'effet demeurait généralement la même après une correction complète et la taille de l'échantillon utilisé par nos analyses fournissait une puissance statistique adéquate. Finalement, certains déterminants pertinents, comme l'obésité ${ }^{22,80}$, n'étaient pas accessibles pour notre étude ${ }^{81}$.

\section{Conclusion}

Nous avons constaté que les risques démographiques et ceux liés à la santé pour l'obtention d'un résultat positif à un test de dépistage du SRAS-CoV-2, qui ont généralement constitué les cibles de la lutte stratégique contre la COVID-19 jusqu'à maintenant, semblent sujets aux biais collisionneurs. Cependant, nous avons observé une corrélation cohérente entre les résultats positifs au test de dépistage du SRAS-CoV-2 et des déterminants sociaux de la santé importants, comme le statut de travailleur essentiel, le nombre de personnes résidant au sein d'un ménage et le niveau de scolarité. Une lutte efficace contre la COVID-19 nécessite que les déterminants sociaux associés à l'accès à un test de dépistage et les risques de transmission du SRAS-CoV-2 soient caractérisés et qu'on s'y attarde à l'aide d'interventions adaptées au risque et ancrées dans la communauté. 


\section{Références}

1. Mishra S, Kwong JC, Chan AK, et al. Understanding hetergeneity to inform public health response to COVID-19 in Canada. CMAJ 2020;192:E684-5.

2. Report of the WHO-China joint mission on coronavirus disease 2019 (COVID-19). Geneva: World Health Organization; 2020.

3. Rosenberg ES, Dufort EM, Blog DS, et al.; New York State Coronavirus 2019 Response Team. COVID-19 testing, epidemic features, hospital outcomes, and household prevalence, New York State: March 2020. Clin Infect Dis 2020;71: 1953-9.

4. Jing Q-L, Liu M-J, Yuan J, et al. Household secondary attack rate of COVID-19 and associated determinants [prépublication]. MedRxiv 2020 Apr. 15. doi: 10.1101/ 2020.04.11.20056010.

5. Bhala N, Curry G, Martineau AR, et al. Sharpening the global focus on ethnicity and race in the time of COVID-19. Lancet 2020;395:1673-6.

6. Chung H, Fung K, Ferreira-Legere LE, et al. COVID-19 Laboratory testing in Ontario: patterns of testing and characteristics of individuals tested, as of April 30, 2020. Toronto: ICES; 2020.

7. Wang L, Ma H, Yiu KCY, et al. Heterogeneity in risk, testing and outcome of COVID-19 across outbreak settings in the Greater Toronto Area, Canada: an observational study. CMAJ Open 2020;8:E627-36.

8. Price-Haywood EG, Burton J, Fort D, et al. Hospitalization and mortality among black patients and white patients with COVID-19. N Engl J Med 2020; 382:2534-43.

9. Yehia BR, Winegar A, Fogel R, et al. Association of race with mortality among patients hospitalized with coronavirus disease 2019 (COVID-19) at 92 US hospitals. JAMA Netw Open 2020;3:e2018039.

10. Feng C, Li J, Sun W, et al. Impact of ambient fine particulate matter (PM2.5) exposure on the risk of influenza-like-illness: a time-series analysis in Beijing, China. Environ Health 2016;15:17.

11. Zoran MA, Savastru RS, Savastru DM, et al. Assessing the relationship between surface levels of PM2.5 and PM10 particulate matter impact on COVID-19 in Milan, Italy. Sci Total Environ 2020;738:139825.

12. Wu X, Nethery RC, Sabath BM, et al. Exposure to air pollution and COVID-19 mortality in the United States: a nationwide cross-sectional study. Sci Adv 2020;6:eabd4049.

13. Liang D, Shi L, Zhao J, et al. Urban air pollution may enhance COVID-19 casefatality and mortality rates in the United States. Innovation (NY) 2020;1:100047.

14. The Lancet Planetary Health. Environmental racism: time to tackle social injustice. Lancet Planet Health 2018;2:e462.

15. Stall NM, Wu W, Lapointe-Shaw L, et al. Sex- and age-specific differences in COVID-19 testing, cases and outcomes: a population-wide study in Ontario, Canada. J Am Geriatr Soc 2020;68:2188-91.

16. Niedzwiedz CL, O'Donnell CA, Jani BD, et al. Ethnic and socioeconomic differences in SARS-CoV-2 infection: prospective cohort study using UK Biobank. BMC Med 2020;18:160.

17. Griffith GJ, Morris TT, Tudball MJ, et al. Collider bias undermines our understanding of COVID-19 disease risk and severity. Nat Commun 2020;11:5749.

18. Hernán MA, Robins JM. Causal inference: what if. Boca Raton (FL): Chapman \& Hall/CRC; 2020

19. Elwert F, Winship C. Endogenous selection bias: the problem of conditioning on a collider variable. Annu Rev Sociol 2014;40:31-53.

20. Hussain A, Bhowmik B, do Vale Moreira NC. COVID-19 and diabetes: knowledge in progress. Diabetes Res Clin Pract 2020;162:108142.

21. Kumar A, Arora A, Sharma P, et al. Is diabetes mellitus associated with mortality and severity of COVID-19? A meta-analysis. Diabetes Metab Syndr 2020;14: 535-45.
22. Palaiodimos L, Kokkinidis DG, Li W, et al. Severe obesity, increasing age and male sex are independently associated with worse in-hospital outcomes, and higher in-hospital mortality, in a cohort of patients with COVID-19 in the Bronx, New York. Metabolism 2020;108:154262.

23. Health care in Ontario: OHIP. Toronto: Government of Ontario. Accessible ici : www.ontario.ca/page/health-care-ontario (consulté le 29 oct. 2020).

24. Schedule of benefits for laboratory services. Toronto: Ministry of Health, Ontario Health Insurance Plan, Laboratories and Genetics Branch; 2020. Accessible ici : www.health.gov.on.ca/en/pro/programs/ohip/sob/lab/lab_mn2020. pdf (consulté le 12 avril 2021).

25. ICES. Data, Discovery, Better Health. Toronto: ICES; 2020. Accessible ici : www. ices.on.ca/Data-and-Privacy/ICES-data/Types-of-ICES-Data (consulté le 29 oct. 2020).

26. Data products, 2016 Census. Ottawa: Statistics Canada; modified 2021 Feb. 8 Accessible ici : www12.statcan.gc.ca/census-recensement/2016/dp-pd/index -eng.cfm (consulté le 29 oct. 2020).

27. Pennington E. Asthma increases risk of severity of COVID-19. Cleve Clin J Med 2020 May 5 [cyberpublication avant impression]. doi: 10.3949/ccjm.87a.ccc002.

28. Williamson EJ, Walker AJ, Bhaskaran K, et al. OpenSAFELY: factors associated with COVID-19 death in 17 million patients. Nature 2020;584:430-6.

29. Fang L, Karakiulakis G, Roth M. Are patients with hyperetension and diabetes mellitus at increased risk for COVID-19 infection? Lancet Respir Med 2020;8:e21.

30. Merkler AE, Parikh NS, Mir S, et al. Risk of ischemic stroke in patients with COVID-19 versus patients with influenza [préimpression]. medRxiv 2020 May 21. doi: 10.1101/2020.05.18.20105494.

31. COVID-19 provincial testing guidance update. V. 4.0, May 14, 2020. Toronto: Ministries of Health and Long-Term Care.

32. COVID-19 provincial testing guidance update: May 2, 2020. Toronto: Ministries of Health and Long-Term Care.

33. COVID-19 provincial testing guidance update: April 15, 2020. Toronto: Ministries of Health and Long-Term Care.

34. COVID-19 provincial testing guidance update: May 28, 2020. Toronto: Ministries of Health and Long-Term Care.

35. COVID-19 quick reference public health guidance on testing and clearance. Toronto: Ministries of Health and Long-Term Care; 2020.

36. COVID-19 provincial testing guidance update: April 8, 2020. Toronto: Ministries of Health and Long-Term Care.

37. Tolea MI, Morris JC, Galvin JE. Trajectory of mobility decline by type of dementia. Alzheimer Dis Assoc Disord 2016;30:60-6.

38. Chiao C-Y, Wu H-S, Hsiao C-Y. Caregiver burden for informal caregivers of patients with dementia: a systematic review. Int Nurs Rev 2015;62:340-50.

39. The Johns Hopkins ACG system. Baltimore: Johns Hopkins University. Accessible ici : www.hopkinsacg.org/ (consulté le $1^{\mathrm{er}}$ nov. 2020)

40. Austin PC, van Walraven C, Wodchis WP, et al. Using the Johns Hopkins Aggre gated Diagnosis Groups (ADGs) to predict mortality in a general adult population cohort in Ontario, Canada. Med Care 2011;49:932-9.

41. van Donkelaar A, Martin RV, Brauer M, et al. Use of satellite observations fo long-term exposure assessment of global concentrations of fine particulate matter. Environ Health Perspect 2015;123:135-43.

42. Hystad $\mathrm{P}$, Setton $\mathrm{E}$, Cervantes $\mathrm{A}$, et al. Creating national air pollution models for population exposure assessment in Canada. Environ Health Perspect 2011;119: $1123-9$

43. Walker PGT, Whittaker C, Watson OJ, et al. The impact of COVID-19 and strategies for mitigation and suppression in low- and middle-income countries. Science 2020;369:413-22. 
44. Park SY, Kim Y-M, Yi S, et al. Coronavirus disease outbreak in call center, South Korea. Emerg Infect Dis 2020;26:1666-70.

45. Chief Public Health Officer of Canada. From risk to resilience: an equity approach to COVID-19. Ottawa: Public Health Agency of Canada; 2020.

46. Karaye IM, Horney JA. The impact of social vulnerability on COVID-19 in the U.S.: an analysis of spatially varying relationships. Am J Prev Med 2020;59: 317-25.

47. Rodriguez-Diaz CE, Guilamo-Ramos V, Mena L, et al. Risk for COVID-19 infection and death among Latinos in the United States: examining heterogeneity in transmission dynamics. Ann Epidemiol 2020;52:46-53.e2.

48. Dictionary, Census of Population, 2016: dissemination area (DA). Ottawa: Statistics Canada; 2016, modified 2019 Jan. 3. Accessible ici : www12.statcan .gc.ca/census-recensement/2016/ref/dict/geo021-eng.cfm (consulté le 13 oct. 2020).

49. Public health units. Toronto: Government of Ontario; 2014, updated 2019 Mar. 22. Accessible ici : www.ontario.ca/page/public-health-units (consulté le 29 oct. 2020)

50. Enhanced epidemiological summary: COVID-19 in Ontario - a focus on diversity. Toronto: Public Health Ontario; 2020.

51. Sullivan SG, Tchetgen EJ, Cowling BJ. Theoretical basis of the test-negative study design for assessment of influenza vaccine effectiveness. Am J Epidemiol 2016;184:345-53.

52. NAAQS Table. Washington (DC): United States Environmental Protection Agency. Accessible ici : www.epa.gov/criteria-air-pollutants/naaqs-table (consulté le 21 sept. 2020).

53. Nishiga M, Wang DW, Han Y, et al. COVID-19 and cardiovascular disease: from basic mechanisms to clinical perspectives. Nat Rev Cardiol 2020;17:543-58.

54. Elliot AJ, Harcourt SE, Hughes HE, et al. The COVID-19 pandemic: a new challenge for syndromic surveillance. Epidemiol Infect 2020;148:e122.

55. Morelli X, Rieux C, Cyrys J, et al. Air pollution, health and social deprivation: a fine-scale risk assessment. Environ Res 2016;147:59-70.

56. Næss O, Piro FN, Nafstad P, et al. Air pollution, social deprivation, and mortality. Epidemiology 2007;18:686-94.

57. Lin SL. Intersectionality and inequalities in medical risk for severe COVID-19 in the Canadian Longitudinal Study on Aging. Gerontologist 2020 Sept. 24 [cyberpublication avant impression]. doi: 10.1093/geront/gnaa143.

58. Drefahl S, Wallace M, Mussino E, et al. A population-based cohort study of socio-demographic risk factors for COVID-19 deaths in Sweden. Nat Commun 2020;11:5097.

59. Luo L, Liu D, Liao X, et al. Contact settings and risk for transmission in 3410 close contacts of patients with COVID-19 in Guangzhou, China: a prospective cohort study. Ann Intern Med 2020;173:879-87.

60. Jing Q-L, Liu M-J, Zhang Z-B, et al. Household secondary attack rate of COVID19 and associated determinants in Guangzhou, China: a retrospective cohort study. Lancet Infect Dis 2020;20:1141-50.

61. Workplace safety \& prevention services guidance on health and safety for the personal care service sector during COVID-19. Mississauga (ON): Workplace Safety \& Prevention Services; 2020.

62. Dyal JW, Grant MP, Broadwater K, et al. COVID-19 among workers in meat and poultry processing facilities: 19 states, April 2020. MMWR Morb Mortal Wkly Rep 2020;69:557-61.

63. Contrera J. The N95 shortage America can't seem to fix. The Washington Post 2020 Sept. 21. Accessible ici : www.washingtonpost.com/graphics/2020/local/ news/n-95-shortage-covid/ (consulté le 21 sept. 2020).
64. Vahidy FS, Nicolas JC, Meeks JR, et al. Racial and ethnic disparities in SARSCoV-2 pandemic: analysis of a COVID-19 observational registry for a diverse US metropolitan population. BMJ Open 2020;10:e039849.

65. de Lusignan S, Dorward J, Correa A, et al. Risk factors for SARS-CoV-2 among patients in the Oxford Royal College of General Practitioners Research and Surveillance Centre primary care network: a cross-sectional study. Lancet Infect Dis 2020;20:1034-42.

66. Liem A, Wang C, Wariyanti $\mathrm{Y}$, et al. The neglected health of international migrant workers in the COVID-19 epidemic. Lancet Psychiatry 2020;7:e20.

67. Ordóñez CE. Not race, racism: concerns of COVID-19 affecting African Americans. Anthropological Responses to Heatlh Emergencies; 2020.

68. Dodds C, Fakoya I. COVID-19: ensuring equality of access to testing for ethnic minorities. BMJ 2020;369:m2122.

69. Brandén M, Aradhya S, Kolk M, et al. Residential context and COVID-19 mortality among adults aged 70 years and older in Stockholm: a population-based, observational study using individual-level data. Lancet Healthy Longev 2020;1: e80-8.

70. Jordan-Martin NC, Madad S, Alves L, et al. Isolation hotels: a community-based intervention to mitigate the spread of the COVID-19 pandemic. Health Secur 2020 Sept. 25 [cyberpublication avant impression]. doi: 10.1089/hs.2020.0123.

71. Madad S, Nuzzo JB, Bordeaux M. The missing piece in America's COVID-19 isolation and quarantine strategy: wraparound services [blog]. Health Affairs 2020 Dec. 10. Accessible ici : www.healthaffairs.org/do/10.1377/hblog20201207 $.458415 /$ full/ (consulté le 9 mars 2021).

72. Vazquez J, Islam T, Beller J, et al. Expanding paid sick leave as a public health tool in the COVID-19 pandemic. J Occup Environ Med 2020;62:e598-9.

73. Rapid COVID-19 testing a potential game-changer in worker protection. Toronto: Institute for Work and Health; 2020. Accessible ici : www.iwh.on.ca/newsletters/at -work/102/rapid-covid-19-testing-potential-game-changer-in-worker-protection (consulté le 9 mars 2021).

74. Webb Hooper M, Napoles AM, Perez-Stable EJ. COVID-19 and racial/ethnic disparities. JAMA 2020;323:2466-7.

75. Yang T-C, Choi S-WE, Sun F. COVID-19 cases in US counties: roles of racial/ethnic density and residential segregation. Ethn Health 2021;26:11-21.

76. Wallis G, Siracusa F, Blank M, et al. Experience of a novel community testing programme for COVID-19 in London: lessons learnt. Clin Med (Lond) 2020;20: e165-9.

77. Good Neighbour Project [page d'accueil]. Accessible ici : www. goodneighbourproject.com (consulté le 22 févr. 2021).

78. Marwaha S, Vohra-Miller S, Grewal R. We started the South Asian COVID Task Force because Ontario failed to address inequities. In a short time, we've seen more people get tested. Toronto Star 2020 Dec. 17. Accessible ici : www.thestar .com/opinion/contributors/2020/12/15/we-started-the-south-asian-covid-task -force-because-ontario-failed-to-address-inequities-in-a-short-time-weve-been -able-to-make-great-strides.html (consulté le 22 févr. 2021).

79. Jabakhanji S. Meet the Toronto Indigenous organizations bringing COVID-19 testing, food directly to people's doorsteps. CBC News Toronto 2020 Dec. 19. Accessible ici : www.cbc.ca/news/canada/toronto/anishnawbe-health-toronto -mobile-unit-covid19-1.5841393 (consulté le 22 févr. 2021).

80. Kwok S, Adam S, Ho JH, et al. Obesity: a critical risk factor in the COVID-19 pandemic. Clin Obes 2020;10:e12403.

81. ICES Data Dictionary. Toronto: ICES. Accessible ici : www.ices.on.ca/Data-and -Privacy/ICES-data/Data-dictionary (consulté le $1^{\mathrm{er}}$ nov. 2020). 
Intérêts concurrents : Adrienne Chan est membre du conseil de Partners in Health Canada. Mackenzie Hamilton est actuellement en stage chez AstraZeneca Canada afin de soutenir les initiatives de recherche en santé en lien avec le lupus et la forme grave de l'asthme. Aucun autre intérêt concurrent n'a été déclaré.

Cet article a été révisé par des pairs.

Affiliations : ICES Central (Sundaram, Calzavara, Hamilton, Djebli, Rosella, Watson, H. Chen, B. Chen, Kwong); Département de médecine (Mishra, Chan); Institut de gestion, d'évaluation et de politiques de santé (Mishra, Chan); Institut des sciences médicales (Mishra); École de santé publique Dalla Lana (Kustra, Chan, Hamilton, Djebli, Rosella, Watson, H. Chen, Kwong); Département des sciences statistiques (Kustra); Département de médecine familiale et communautaire (Kwong), Université de Toronto; MAP Centre for Urban Health Solutions (Mishra), Institut du savoir Li Ka Shing, Hôpital St. Michael, Unity Health Toronto; Centre des sciences de la santé Sunnybrook (Chan); Santé publique Ontario (Kwong, H. Chen); Toronto, Ont.; Département d'épidémiologie (Baral), École de santé publique Bloomberg de l'Université Johns Hopkins, Baltimore, Md.

Collaborateurs : Jeffrey Kwong, Sharmistha Mishra et Stefan Baral ont conçu l'étude. Andrew Calzavara a réalisé l'ensemble des analyses de données (création de l'ensemble de données et de variables et modélisation statistique). Jeffrey Kwong, Sharmistha Mishra, Stefan Baral, Rafal Kustra et Andrew Calzavara ont conçu les plans d'analyse et réalisé la sélection des variables, avec la contribution de Hong Chen et Adrienne Chan pour la sélection et la définition des variables. Mackenzie Hamilton, Mohamed Djebli, Laura Rosella et Tristan Watson ont contribué aux plans d'analyse en lien avec le biais collisionneur. Branson Chen a contribué aux analyses et à la préparation des données pour l'ensemble des données symptomatiques. Maria Sundaram, Jeffrey Kwong, Stefan Baral et Sharmistha Mishra ont rédigé le manuscrit. Tous les auteurs ont contribué à l'interprétation des données, ont révisé de façon critique le contenu intellectuel important du manuscrit, ont donné leur approbation finale pour la version destinée à être publiée et endossent l'entière responsabilité de tous les aspects du travail. Sharmistha Mishra et Stefan Baral sont les auteurs principaux.

Propriété intellectuelle du contenu : Il s'agit d'un article en libre accès distribué conformément aux modalités de la licence Creative Commons Attribution (CC BY-NC-ND 4.0), qui permet l'utilisation, la diffusion et la reproduction de tout médium à la condition que la publication originale soit adéquatement citée, que l'utilisation se fasse à des fins non commerciales (c.-à-d., recherche ou éducation) et qu'aucune modification ni adaptation n'y soit apportée. Voir : https://creativecommons.org/licenses/by-nc-nd/4.0/deed.fr.
Soutien financier : La présente étude a été financée par une subvention de recherche sur la COVID-19 du Conseil de l'innovation en recherche de l'Hôpital St. Michael. Stefan Baral, Jeffery Kwong, Sharmistha Mishra et Maria Sundaram ont obtenu une subvention de fonctionnement en recherche (VR5-172683) des Instituts de recherche en santé du Canada. Sharmistha Mishra est soutenue par une chaire de recherche du Canada en science de la modélisation mathématique et programmation de niveau 2. Jeffrey Kwong est soutenu par une bourse de clinicien-chercheur du Département de médecine familiale et communautaire de l'Université de Toronto.

Partage des données : L'ensemble de données de la présente étude a été codé et est hébergé de façon sécuritaire par l'ICES. Bien que des ententes sur le partage des données entre l'ICES et les fournisseurs de données (p. ex., les organismes de soins de santé et les instances gouvernementales) empêchent l'ICES de rendre les données accessibles au public, l'accès peut être accordé aux personnes répondant aux critères d'accès confidentiels préétablis, accessibles ici : https://www.ices.on. ca/DAS (courriel : das@ices.on.ca). Le plan de création des données et le code analytique sous-jacent seront fournis sur demande par les auteurs. À noter que certains programmes informatiques pourraient reposer sur des modèles de codage ou des macros uniques à l'ICES et par conséquent, inaccessibles ou nécessitant des modifications.

Remerciements : Les auteurs remercient IQVIA Solutions Canada pour l'utilisation de leur Fichier de renseignement sur les médicaments, de même que Owen Langman pour son soutien technique. Finalement, les auteurs remercient les 14,7 millions résidents de l'Ontario sans lesquels ce projet de recherche n'aurait pas été possible.

Avis de non-responsabilité : Les opinions, résultats et conclusions présentés dans cet article n'engagent que ses auteurs et sont indépendants des sources de financement. Une partie du présent document est fondé sur des données ou des renseignements compilés et fournis par I'Institut canadien d'information sur la santé (ICIS) et Action Cancer Ontario (ACO). Toutefois, les analyses, les conclusions, les opinions et les énoncés exprimés aux présentes n'engagent que les auteurs et non l'ICIS et l'ACO. Aucun appui de la part de l'ICES, du ministère de la Santé et des Soins de longue durée (MSSLD) de l'Ontario, de l'ICIS ou de ACO n'est sous-entendu ni ne devrait être inféré.

Cette étude a bénéficié du soutien de l'ICES, qui reçoit une subvention annuelle du ministère de la Santé et des Soins de longue durée (MSSLD) de l'Ontario. Les commanditaires de l'étude n'ont pas participé à la conception et à la mise en œuvre de l'étude, ni à la collecte, la gestion, l'analyse et l'interprétation des données, ni à la préparation, la révision ou l'approbation du manuscrit, ni à la décision de soumettre le manuscrit pour publication.

Accepté : Le 6 avril 2021

Correspondance : Jeffrey Kwong, jeff.kwong@utoronto.ca 